\title{
Distributed Brain Co-Processor for Neurophysiologic Tracking and Adap- tive Stimulation: Application to Drug Resistant Epilepsy
}

Authors:

Vladimir Sladky ${ }^{1,2,13 \dagger}$, Petr Nejedly ${ }^{1,2,15 \dagger}$, Filip Mivalt ${ }^{1,14}$, Benjamin H. Brinkmann ${ }^{1,3}$, Inyong Kim ${ }^{1}$, Erik K. St. Louis ${ }^{4}$, Nicholas M. Gregg ${ }^{1}$, Brian N. Lundstrom ${ }^{1}$, Chelsea M. Crowe ${ }^{5}$, Tal Pal Attia ${ }^{1}$, Daniel Crepeau ${ }^{1}$, Irena Balzekas ${ }^{1,16,17}$, Victoria Marks ${ }^{1,16}$, Lydia P. Wheeler ${ }^{1,16}$, Jan Cimbalnik ${ }^{2}$, Mark Cook $^{6}$, Radek Janca ${ }^{11}$, Beverly K. Sturges ${ }^{5}$, Kent Leyde ${ }^{7}$, Kai J. Miller ${ }^{10}$, Jamie J. Van Gompel ${ }^{10}$, Timothy Denison ${ }^{9}$, Gregory A. Worrell ${ }^{1,3 * *}$, Vaclav Kremen ${ }^{1,12 * *}$

\section{Affiliations:}

${ }^{1}$ Department of Neurology, Bioelectronics Neurophysiology and Engineering Laboratory, Mayo Clinic, Rochester, MN, USA.

${ }^{2}$ International Clinical Research Center, St. Anne's University Hospital, Brno, Czech Republic.

${ }^{3}$ Department of Physiology and Biomedical Engineering, Mayo Clinic, Rochester, MN, USA.

${ }^{4}$ Center for Sleep Medicine, Departments of Neurology and Medicine, Divisions of Sleep Neurology \& Pulmonary and Critical Care Medicine, Mayo Clinic, Rochester, MN.

${ }^{5}$ Department of Veterinary Clinical Sciences, University of California, Davis, CA, USA.

${ }^{6}$ Department of Neurology, Royal Melbourne Hospital, Melbourne, Australia.

${ }^{7}$ Cadence Neuroscience, Seattle, WA.

${ }^{8}$ Medtronic PLC, Minneapolis, MN.

${ }^{9}$ Department of Bioengineering, Oxford University, Oxford, UK.

${ }^{10}$ Department of Neurologic Surgery, Mayo Clinic, Rochester, MN, USA.

${ }^{11}$ Faculty of Electrical Engineering, Czech Technical University in Prague, Prague, Czech Republic.

${ }^{12}$ Czech Institute of Informatics, Robotics, and Cybernetics, Czech Technical University in Prague, Prague, Czech Republic.

${ }^{13}$ Faculty of Biomedical Engineering, Czech Technical University in Prague, Kladno, Czech Republic.

${ }^{14}$ Faculty of Electrical Engineering and Communication, Brno University of Technology, Brno, Czech Republic.

${ }^{15}$ The Czech Academy of Sciences, Institute of Scientific Instruments, Brno, Czech Republic.

${ }^{16}$ Biomedical Engineering and Physiology Graduate Program, Mayo Clinic Graduate School of Biomedical Sciences.

${ }^{17}$ Mayo Clinic School of Medicine and the Mayo Clinic Medical Scientist Training Program.

${ }^{\dagger}$ authors contributed equally

* senior and corresponding authors (worrell.gregory@mayo.edu \& kremen.vaclav@mayo.edu)

\section{One Sentence Summary:}

We created a distributed brain co-processor for continuous neurophysiologic tracking and controlling adaptive brain stimulation to treat epilepsy. 
Abstract: Electrical brain stimulation is a proven therapy for epilepsy, but long-term seizure free outcomes are rare. Early implantable devices were developed for open-loop stimulation without sensing, embedded computing or adaptive therapy. Recent device advances include sensing and closed-loop responsive stimulation, but these clinically available devices lack adequate computing, data storage and patient interface to concisely catalog behavior, seizures, and brain electrophysiology, despite the critical importance of these details for epilepsy management. Here we describe the first application of a distributed brain co-processor providing an intuitive, bi-directional interface between device implant, patient \& physician, and implement it in human and canine patients with epilepsy living in their natural environments. Automated behavioral state tracking (awake and sleep) and electrophysiologic classifiers for interictal epileptiform discharges and electrographic seizures are run on local hand-held and distributed cloud computing resources to guide adaptive electrical stimulation. These algorithms were first developed and parameterized using long-term retrospective data from 10 humans and 11 canines with epilepsy and then implemented prospectively in two pet canines and one human with drug resistant epilepsy as they naturally navigate their lives in society. 


\section{INTRODUCTION}

FDA approved electrical brain stimulation (EBS) devices for neurological and psychiatric diseases include responsive neural stimulation (RNS) for epilepsy (1-3) and deep brain stimulation (DBS) for epilepsy (4, 5), dystonia (6), Parkinson's disease (7), essential tremor (8), and obsessive-compulsive disorder (humanitarian device exemption) (9). Emerging EBS applications include depression (1012), cognitive enhancement $(13,14)$ and Alzheimer's disease (15-17). While open-loop $(5,11,12$, 18) and responsive closed-loop $(2,3)$ stimulation with pre-programmed parameters of high or low frequency stimulation have been successful in reducing seizures, there remains intense interest in refinement of neural stimulation by development of adaptive closed-loop EBS, where the vast range of possible stimulation parameters can be adaptively optimized for individualized therapy (19-21). There remain, however, basic technology gaps to adaptive therapy with inadequate device computing, data storage and patient interface capabilities required to accurately catalog and track behavior, seizures, and brain electrophysiology in ambulatory subjects in naturalistic settings.

Here we describe a distributed brain co-processor for integration of implantable brain sensing and stimulation devices with off-the-body commercial electronics for clinical and neuroscience research applications (21-23). The integration of implantable devices with commercial electronics allows computational power and algorithm complexity to increase with advances in consumer computer hardware, without requiring surgical replacement of implanted components. Brain implants providing bi-directional wireless connectivity with a smartphone and distributed cloud computing overcome the computational and data storage limitations of current implantable devices. Until recently, there have been obstacles to consolidating the multiple-technology layers required for continuous brain tracking, behavior reporting, and adaptive closed-loop therapy. Here we demonstrate these capabilities, utilizing the investigational Medtronic Summit $R C+S^{T M}\left(R C+S^{T M}\right)$ a rechargeable, sensing and stimulation implantable device with a bi-directional application programming interface (21-24). The system enables continuous brain electrophysiology data streaming to a local tablet computer for real-time analysis and tracking of interictal epileptiform spike (IES), seizures, and brain behavioral state coupled with patient reports to inform adaptive EBS therapy for dynamic modulation of the neural circuits underlying focal epilepsy (Fig. 1). The multimodal electrophysiology classifiers (seizure, behavioral state and IES) were validated, tested, and prospectively deployed with adaptive EBS in canine and human subjects with epilepsy.

\section{RESULTS}

\section{Tracking behavior, brain state, and epilepsy biomarkers in humans \& canines}

We used intracranially recorded electroencephalography (iEEG) to classify and track brain states (wake, sleep), seizures and IES rates in ambulatory humans and canines with drug resistant epilepsy living in their natural environments. Continuous streaming iEEG was analyzed on a local handheld computer and cloud using automated classifiers for IES, seizures, and behavioral state that were then reviewed on a custom web-based Epilepsy Dashboard. The system provided an integrated machine learning platform for data viewing, analytics and expert labeling of candidate events, enabling confirmation that a detected electrophysiological event or patient reported event was a true positive seizure (Fig. 1). Physicians remain in the therapy optimization loop using the Epilepsy Dashboard to review trends in biomarker analytics (IES rates), confirmed seizures, sleep/wake hypnograms, patient annotations (reported seizures, medication logs, and mood scores) and implanted device data (battery status, telemetry, and EBS parameters). 

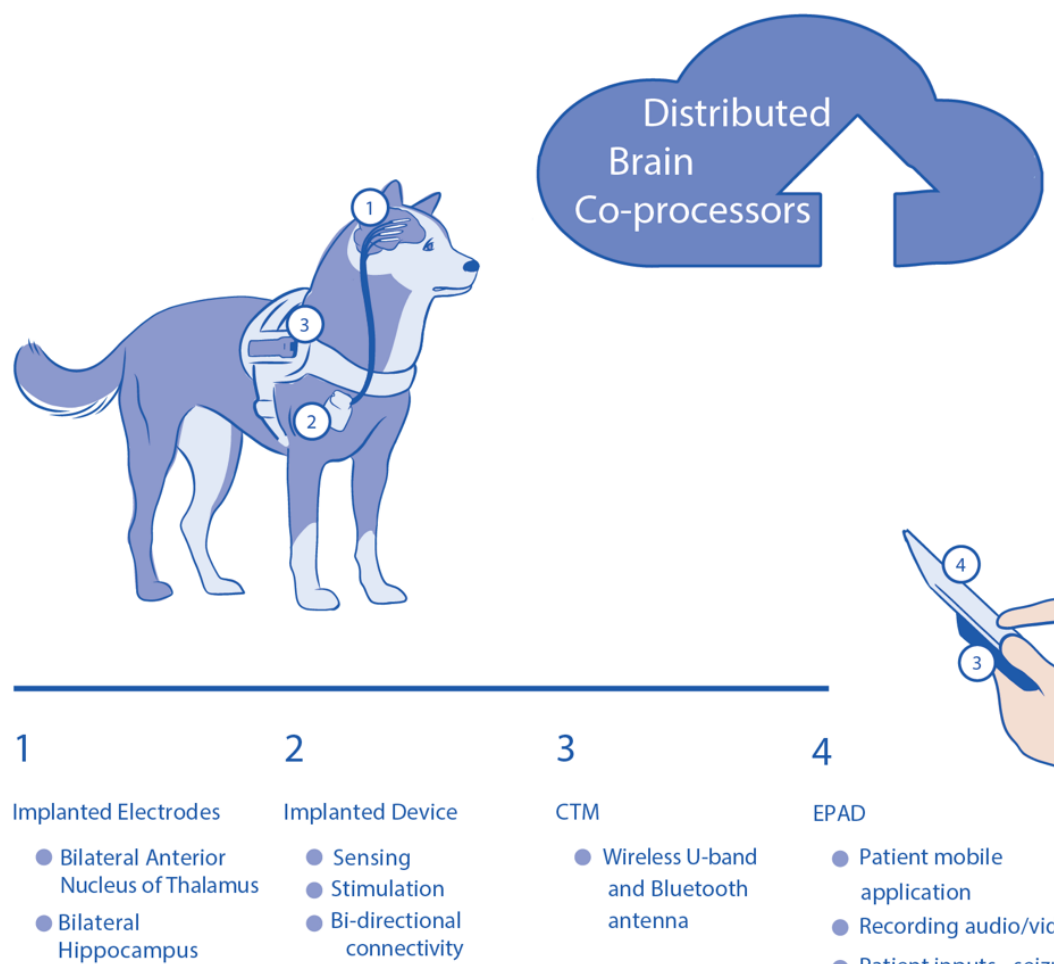

3

СтM

- Wireless U-band and Bluetooth antenna

\section{4}

EPAD

- Patient mobile

application

- Recording audio/video

- Patient inputs - seizure, aura, medication, questionnaires

- The system creates a dense behavioral catalog

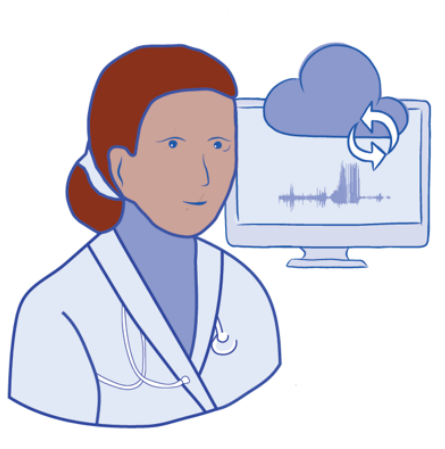

\section{CLOUD DATABASE}

Data and analytics via web interface

- Data curation \& storage

- Data annotation

- Data viewing

- Patient inputs (eg. seizure reports)

- Automated Classification

O Behavioral states

O Interictal epileptiform spikes

O Seizures
SLEEP STAGE

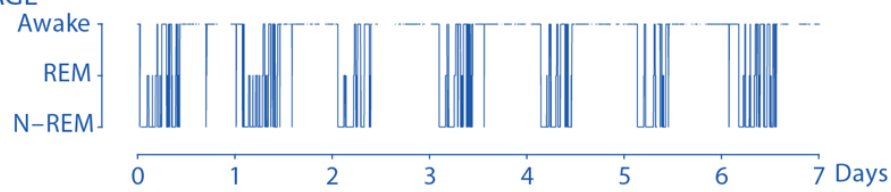

SPIKE RATE 4000

Hippocampus

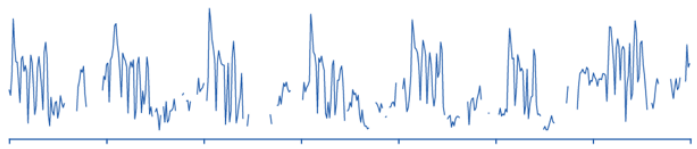

Confirmed seizure after Reviewed event Seizure marked by physician review $[$ (not seizure) $[$ patient

SEIZURE
PROBABILITY

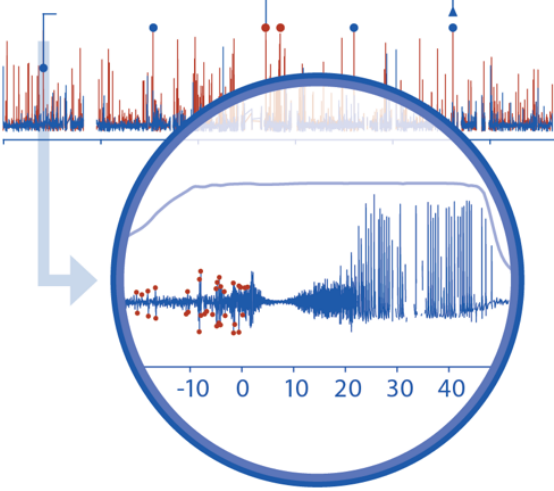

- IEDs

$\mathrm{V}[\mathrm{uV}] 2000$

$-500^{-}$

Time in seconds

Fig. 1. Distributed Brain Co-processor. Integrating implanted sensing and stimulation devices with off-the-body co-processor and cloud computing resources. The system enables continuous brain behavioral state (wake, sleep), seizures, biomarker (interictal epileptiform spikes (IES)) and behavior (patient inputs, actigraphy, mood, memory) tracking, coupled with adaptive electrical stim- 
ulation. The system was developed and prospectively tested in canines and humans with drug resistant epilepsy living in their natural environments. Top) Schematic for bi-directional data transmission (using Clinician Telemetry Module - CTM) between implanted brain sensing and stimulation device integrated with local handheld computer (Epilepsy Patient Assist Device - EPAD) and cloud environment. The integrated system provides a platform for real-time, chronic, remote ambulatory monitoring of patient reported behavior information, device data (battery, telemetry, etc.), brain states, seizures, and biomarkers. Bottom left) The cloud based physician Epilepsy Dashboard provides a platform for review of electrophysiology data that are wirelessly telemetered off the implant and automatically processed with a battery of algorithms running on the patient's local handheld for detecting seizures, IES, and classifying sleep/wake behavioral state. Results are stored into a database and accessible via a web-based dashboard. Bottom right) Epilepsy Dashboard enables swift review of immediate and long-term data trends from the device (e.g., battery, electrode impedances), electrophysiology data, and patient inputs. The physician can quickly review and either confirm or reject automatically detected and patient reported events. The panel shows representative results from 7-days of continuous human hippocampal $(\mathrm{Hc})$ recording with automated classification labels for behavioral state (wake/sleep) IES rates, and seizure detection probability. Blue triangles show patient reported seizure events. Circles in the seizure detection probability graph show automated seizure detections either confirmed as seizures (blue dots) or false positive (red) by expert visual review. The results show that IES rates are increased in NREM sleep phases. Zoomed in plot circular inset shows example of raw data from left $\mathrm{Hc}$ with automatically marked IES (red circles). The patient was aware and reported (blue triangle) of only one seizure out of the six seizures automatically detected in the continuous iEEG and confirmed by the physician.

The development of brain behavioral state, IES, and seizure detection algorithms used training, validation, and pseudo-prospective testing data from a large database of ambulatory iEEG from 11 dogs and 10 human subjects. The final training, validation, and prospective testing of automated algorithms was performed in a human and two pet canines with drug resistant epilepsy over multiple months living in their natural environments.

\section{Automated brain behavioral state classification}

Although the clinical standard for sleep staging involves scalp EEG, we recently demonstrated automated sleep staging using iEEG recordings from humans with epilepsy $(25,26)$. Sleep staging based on iEEG was necessary here for behavioral state classification in ambulatory subjects with implanted sensing devices. Here we extended a previously validated algorithm to human $(\mathrm{MH} 1)$ and canine (MD1-3) subjects (Table 1.) using simultaneous iEEG and scalp EEG, accelerometry, and video recordings to train, validate and test the behavioral state classifier for wake, rapid eye movement (REM) and non-rapid eye movement (NREM - N2 and N3, i.e., slow wave) sleep. Canines are known to have similar sleep architecture to humans, but with more rapid cycling between sleep states (27, 28).

The prospective testing was performed using data from a human subject and three canines and using an algorithm trained, validated, and tested using simultaneous polysomnography and iEEG recordings under different stimulation parameters over multiple days and nights. We used the first night as the training dataset, second night for validation, and third night as testing dataset to confirm the reliability and accuracy of the iEEG based classifier compared to gold standard sleep scoring by visual review of polysomnography (29). Then we prospectively deployed the classifier for continuous ambulatory iEEG based brain state classification in a human (MH1) and two pet canines (UCD1, UCD2) in their natural environments (see below in the Prospective Deployment section). 


\section{$\mathrm{MH} 1$}
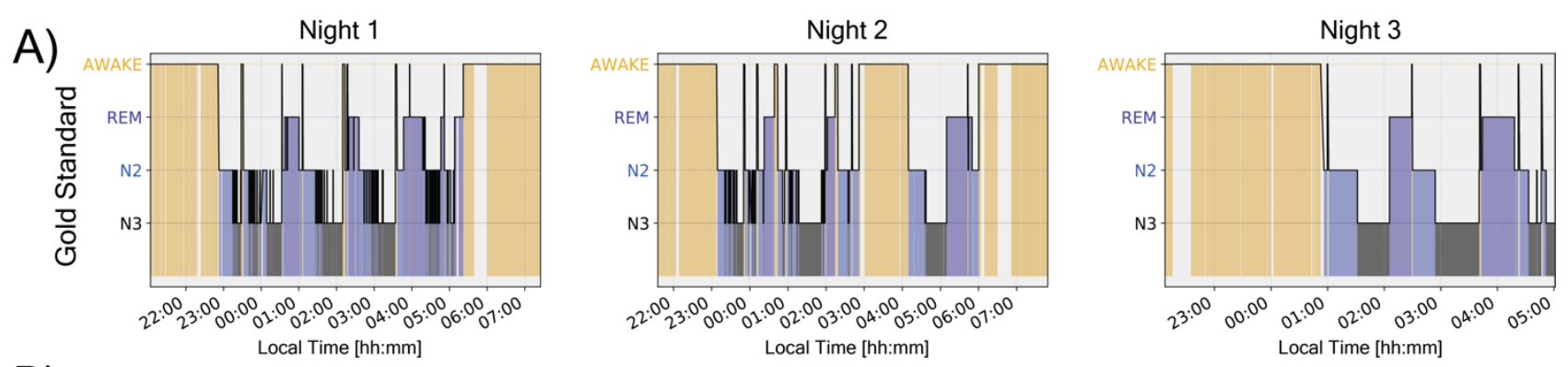

B)
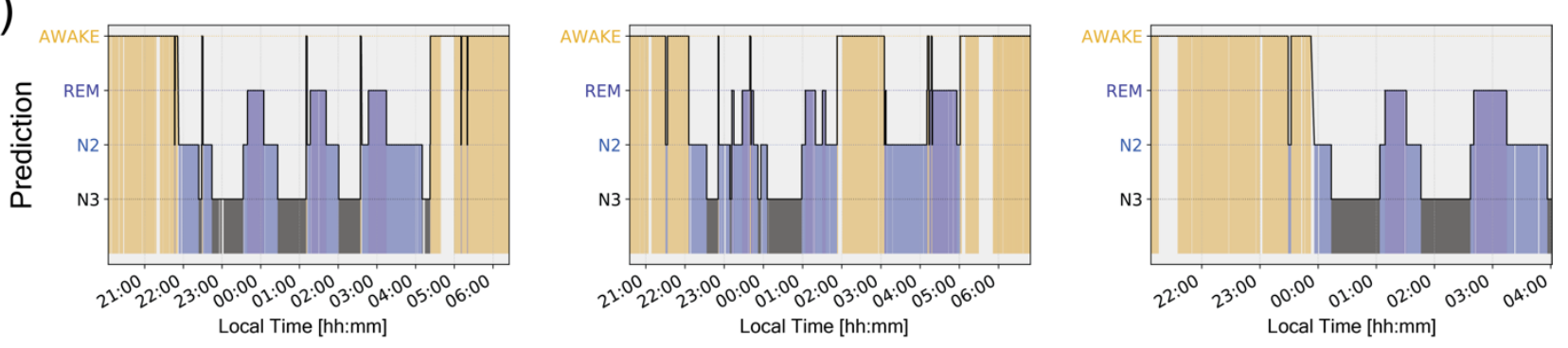

C)
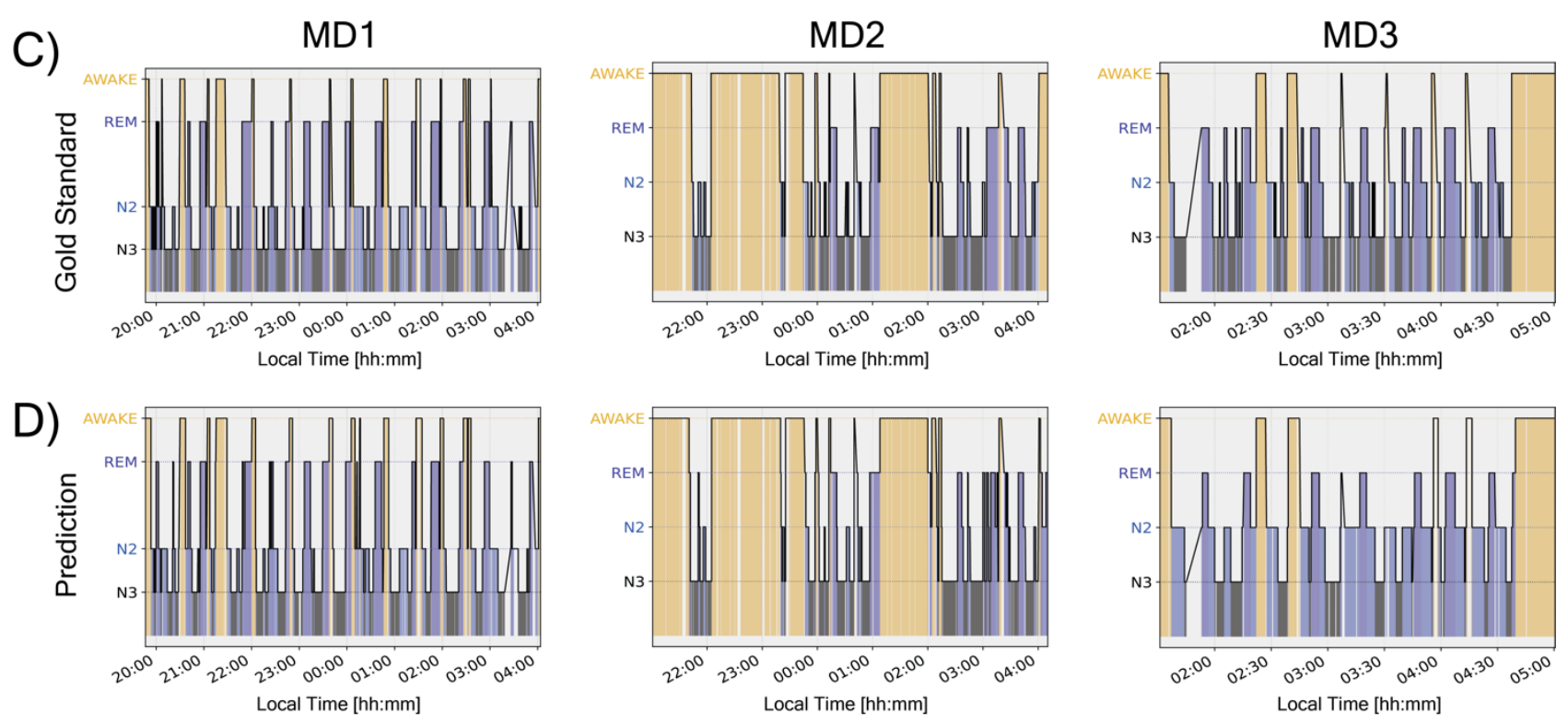

Fig. 2. A visual comparison of sleep scoring. The sleep scoring (hypnograms) from the automated intracranial EEG sleep classifier (bottom) and manual expert review scored sleep classification using scalp polysomnography data in a human (MH1) and three dogs (MD1, 2, 3). (A) The upper panel of hypnograms show brain state (Awake, non-rapid movement (NREM 2, 3) and rapid eye movement (REM) sleep) over three consecutive days/nights using manual review of the polysomnogram (30) for the human subject. (B) Human hypnogram created by the automated sleep classification algorithm. (C) shows the manual scalp sleep scoring of polysomnogram and (D) the automated sleep scoring for three dogs. The number of sleep phases, length of the sleep, and REM latency from the automated classifier is highly concordant with the gold standard expert manual review. The misclassifications generated by the automated classifier primarily occur during transitions between sleep states and fast state changes (like arousals). Table 1. Provides the summary classification performance. 
The automated behavioral state classification method $(25,26)$ uses multiple iEEG power-in-band features and a Naive Bayesian classifier. The algorithm obtained a mean classification accuracy of $81 \%$ and $82 \%$ and weighted average $\mathrm{F} 1$-score $79 \%$ and $82 \%$ for human and canine subjects, respectively. Figure 2 and Table 1 show detailed classification results. In general, the classifier works best for assessing NREM sleep and wake states but achieved acceptable performance for classifying REM sleep. The automated classifier mainly differed from expert-annotated polysomnograms in the transition phases between sleep states. The boundary data create the largest classification errors, as might be expected for this kind of classification task. This automated approach to behavioral state classification, grounded in training data that included clinical standard polysomnography and iEEG, enables long term iEEG-based sleep staging in ambulatory canine and human subjects.

\section{Automated seizure detection}

Accurate seizure catalogues are critical for epilepsy management and assessment of therapeutic outcomes, but remain a basic technology gap for the field $(30,31)$. We created an accurate seizure diary with a generic seizure detector using a Long-Short-Term-Memory (LSTM) (32) artificial recurrent neural network (RNN) coupled with a convolutional neural network (CNN) (33) applied to continuous iEEG to reliably detect spontaneous seizures in ambulatory canine and human subjects with epilepsy.

The large testing, validation, and training dataset from multiple brain structures in humans and canines was collected over multiple years with the two different (NeuroVista Inc. or Medtronic PLC) implantable recording devices (see methods). The LSTM model was trained on a large dataset from dogs with naturally occurring epilepsy ( $n=5$, average 600 days of recording, 340 seizures) implanted with NeuroVista devices $(34,35)$ and one half of the data from two, randomly selected human subjects with epilepsy implanted with the NeuroVista device (total 524 seizures). The model was then validated on the other half of the data from the two NeuroVista patients (524 seizures) and three canines (600 days of recording and 133 spontaneous seizures in dogs: UCD1, UCD2, MD4) implanted with $\mathrm{RC}+\mathrm{S}^{\mathrm{TM}}$ devices (23) (Table 2.).

Two of the dogs implanted with the $R C+S^{T M}$ devices in the validation dataset were pet dogs with epilepsy (UCD1, UCD2) living at home with their owners. The training, validation, and testing schema (Table 2) was used to train the generalizable model for dog and human seizure detection, and subsequently deployed pseudo-prospectively to the rest of the NeuroVista human dataset (7 patients, over 10 years of recording and 2046 seizures) (36). The model was then prospectively deployed and tested in 2 pet canines (UCD1, UCD2) and 1 human subject (MH1) with epilepsy implanted with the $\mathrm{RC}+\mathrm{S}^{\mathrm{TM}}$.

Spontaneous seizures recorded with iEEG show characteristic spectral patterns that are readily identified visually and may be reliably detected by machine learning approaches (37). Figure 3 shows an example of a typical seizure with its time-frequency (spectrogram) characteristics, raw data, and LSTM model seizure probability for $\mathrm{MH} 1$ from the out-of-sample data. Figure 3 also shows how the model probability for seizure classification changes in context of raw iEEG and spectral content and physician gold standard seizure annotation. The figure also illustrates the robustness of the model, showing high probabilities close-to seizure region and low probability outside the seizure (before and after the seizure). The example highlights the importance of LSTM function in the model, since feature-based machine learning models would detect the bursts of IES at the beginning and during the seizure, while the LSTM model raises the seizure probability prior and during the seizure time. 


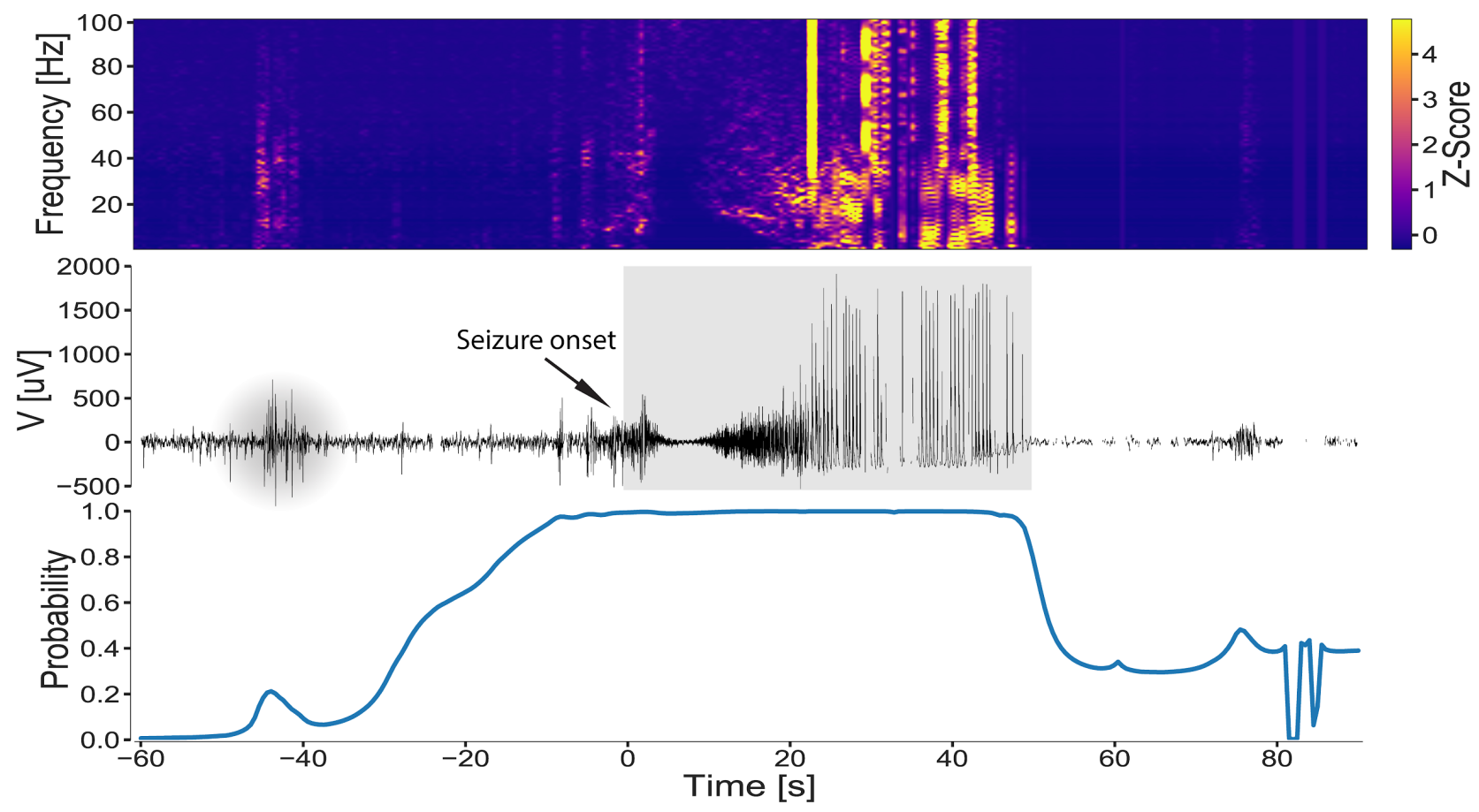

Fig. 3. A representative hippocampal seizure from human subject MH1. Top) Time-frequency characteristics (z-score spectrogram), Middle) Raw intracranial EEG data with the physician annotated (grey highlight) seizure duration, and Bottom) Model seizure probability for patient $\mathrm{MH} 1$ in the out-of-sample dataset demonstrating how the probability of the LSTM model changes over a peri-seizure period of time (pre-ictal, ictal, and post-ictal period). The high probability (near 1) in the peri-seizure region highlights the impact of the long-short-term memory function for raising the probability during and around the seizure time.

The precision recall curves (PRC) and receiver operator curves (ROC) curves are calculated by sequentially changing the model probability threshold and evaluating the results for all seizures from each subject in the testing datasets (Fig. 4). Using the LSTM model, we successfully detected seizures and consequently determined seizure rates over more than 1 year of canine and human recordings, constituting an objective seizure diary. 
A

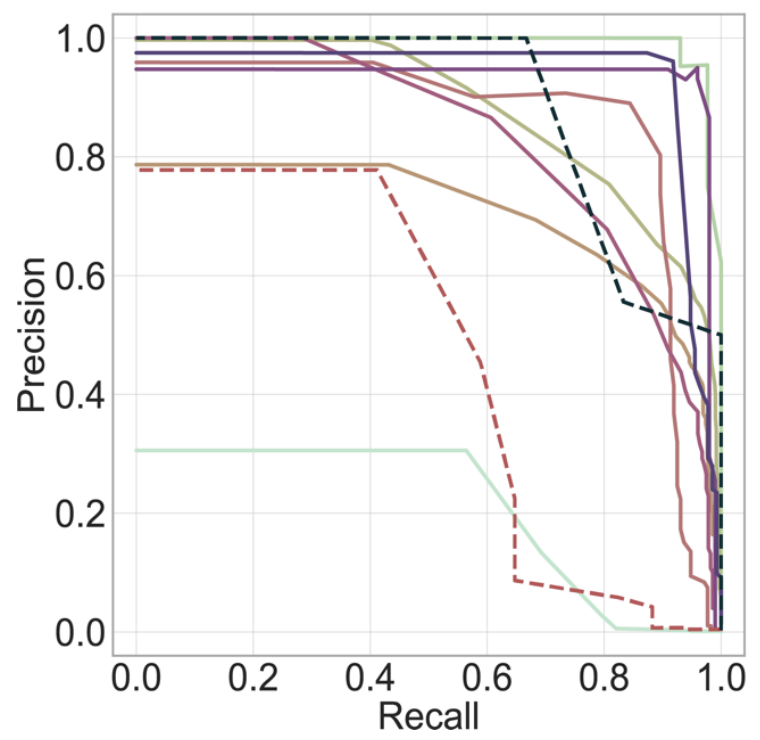

B

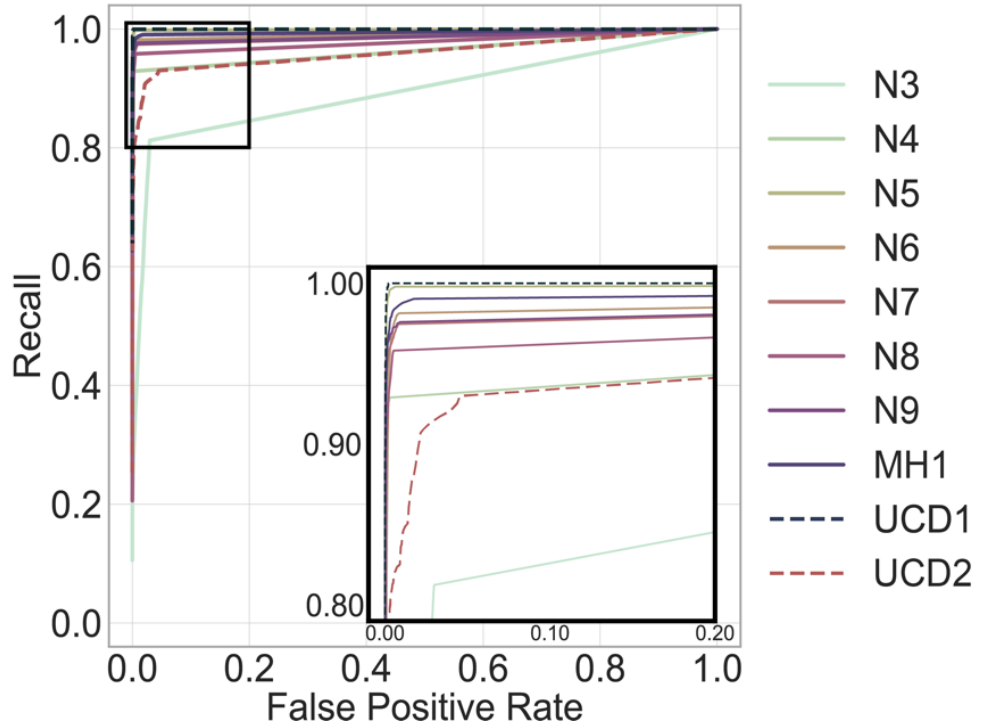

Fig. 4. The long short term memory (LSTM) model performance. Out-of-sample testing in human (solid lines, N3-9 and MH1) and canine (dashed lines) subjects is shown. (A) Precision Recall Curves $(P R C)$ and (B) Receiver Operating Curves (ROC). The detailed view of the left-top square of the ROC shows the results for each subject in the optimal setting of the detector minimizing false positive rate and maximizing sensitivity. The PRC and ROC curves are calculated by sequential changing of the threshold on the model probability and evaluating the results of Precision, Recall, and False Positive Rate for all seizures of each subject in the testing datasets. 


\section{Detection of interictal epileptiform spikes (IES)}

Interictal epileptiform spikes (IES) are an established biomarker of epileptogenic brain (38), and associated with risk for spontaneous, unprovoked seizures (39-41). For long iEEG datasets it is labor intensive and impractical to use visual analysis to calculate IES rates. Here we trained, validated, and tested an automated IES detector on long-term continuous ambulatory iEEG recordings. We implemented a previously published automated IES detection algorithm (42), where the data are continuously accumulated by streaming iEEG from the RC+S ${ }^{T M}$ device to a cloud database. We compared the automated IES detections to expert visual scoring from two epileptologists (NG \& GW) in distinct behavioral states, wake \& N3 sleep. These data included periods of low or high seizure counts during day and night. There was good concordance for the IES labeling by expert visual review (Cohen's kappa score 0.87) and between the algorithm and experts (F1-score $0.82 \pm 0.08$ with sensitivity $91 \pm 0.6 \%$ and positive predictive value $77 \pm 1.6 \%$ ).

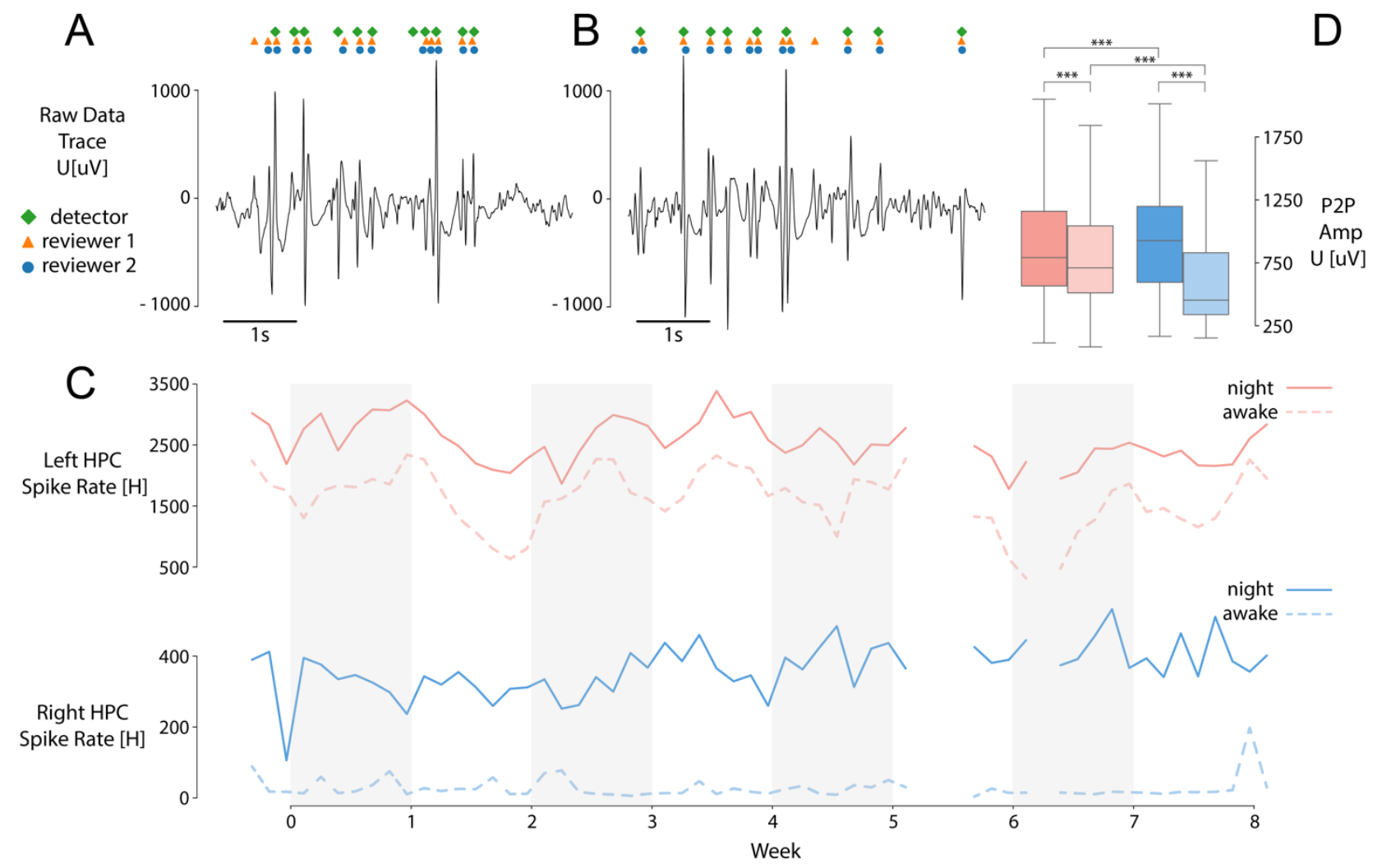

Fig. 5. Long-term analysis of IES rates of MH1. Visual example of comparing spike detections between the automated approach and human operators in (A) day/awake and (B) night/sleep period of time. (C) Daily averaged spike rate per hour in left (top) and right (bottom) hippocampus during night and day periods of time. (D) There are significant differences between night/day and left/right hippocampal IES characteristics (peak-to-peak amplitude) during the two-month period. 
Table 4 summarizes the performance of the IES algorithm in different behavioral states. The algorithm performs well in both seizure cluster periods that have higher IES rates and periods without seizure clusters and lower IES rates (F1-score was 0.84 in seizure cluster and 0.80 in non-cluster periods) (41). Despite the difference in IES rates between day (approximately $25 \%$ lower IES rates) and night the algorithm performed similarly (day F1-score was 0.81 and 0.82 at night). Visual examples of IEDs and comparison of automated detections with expert review are shown in Fig. 5 for day $(A)$ and the night $(B)$ and illustrate the concordance between expert visual review and the automated classifier. The hippocampus IES rate variations during day and night over a two-month period show circadian and multi-day fluctuations (Fig. 5C). We analyzed IES characteristics to explore how the hippocampal IES properties differ in various behavioral states (Fig. 5D) and find higher peak to trough IES amplitudes during night compared to wake $(p<0.001)$.

\section{Prospective long-term ambulatory monitoring and algorithm testing}

We deployed the automated IES and seizure classifiers prospectively in a human and two pet dogs with epilepsy living in their home environments. In the human subject automated brain behavioral state (wake/sleep) classifications were also tracked. Data were prospectively collected over 365 days with 237 seizures recorded in the human subject (MH1). In total, the system was able to record 72 percent of the data (assuming 365*24 hours as 100\%). The patient reported only 56 out of 237 detected, and visually verified seizures. In two pet dogs, prospective data collection spanned over 690 days with continuous data streaming transmitting $49 \%$ of the data on average. The pet owners marked only 43 out of 144 detected and visually verified seizures (Table 6). Because the iEEG is continuously streamed off the implant and stored, we can definitively assess by expert visual review the seizure and IES detection algorithm performance (Table $3 \& 4$ ). The absence of at home polysomnograms during the ambulatory prospective phase preclude further validation of the behavioral state classifier with the clinical gold-standard polysomnogram, although the diurnal patterns of wake and sleep states were qualitatively similar to the hypnograms recorded with polysomnography in the hospital environment.

The prospective testing of the seizure detector in ambulatory subjects in real-world environments showed excellent performance, with an area under the ROC (AUROC) compared to the expert visual review of $0.99,0.96$, and 0.99 for the human (MH1) and the two pet canines (UCD1,2), respectively. The area under the PRC (AUPRC) that more accurately describes the results of this highly imbalanced data was $0.93,0.47$, and 0.88 for the human and two pet canines (UCD1,2), respectively (Table 3.). The performance of the IES detection in the ambulatory prospective data compared to gold standard expert visual reviewed events was 0.9 sensitivity and F1-score of 0.81 .

\section{Electrical brain stimulation (EBS) effect on IES, seizures, and brain behavioral state}

A protocol comparing baseline (no stimulation), low frequency (LF) (2 \& $7 \mathrm{~Hz}$ ), and high frequency (HF) (100 \& $145 \mathrm{~Hz}$ ) stimulation applied to the anterior nucleus of the thalamus (ANT) was used to investigate the effect of ANT EBS on behavioral state, IES, and seizures. In this preliminary, feasibility investigation EBS was applied only to the ANT with continuous iEEG streaming from ANT and hippocampal electrodes. The ANT EBS was shown to modulate behavioral state, IES rates, and seizure rates in human and canine epilepsy. 
In the human subject low, high, and adaptive EBS increased N2 sleep, but reduced REM and total sleep time (Table 5). The patient reported markedly disrupted sleep during continuous HF stimulation with the subjective sense of confusion between reality and dreams that became very distressing with increasing anxiety and declining mood $(43,44)$. Because of the possible role of continuous HF EBS on sleep and mood, we subsequently deployed an adaptive circadian EBS paradigm with a continuous LF stimulation coupled with diurnal responsive HF stimulation targeting seizures and increased IES rates that disabled responsive HF stimulation during sleep. The transition to this adaptive paradigm of continuous LF with responsive HF during wake behavioral state was well tolerated.

Low frequency EBS decreased hippocampal IES rates in both human (MH1) and canine (UCD1 \& UCD2) subjects, and in MH1 and UCD1 modestly reduced seizure counts. High frequency EBS had a more variable effect, reducing IES in the human (MH1) but increasing IES in one dog (UCD1) while modestly decreasing seizure counts.

\section{DISCUSSION}

There has been significant progress in EBS devices for drug resistant epilepsy, but the time to achieve optimal individualized stimulation parameters is long and seizure free outcomes remain rare. The optimal EBS parameters and stimulation paradigm (continuous, duty cycle, responsive, or adaptive) for preventing or stopping focal seizures propagation remains unclear (45). The inability to continuously track electrophysiology, seizure counts, sleep and behavior have impeded systematic investigations of individualized EBS therapy. There is evidence that both low frequency $(<7 \mathrm{~Hz})$ and high frequency $(>100 \mathrm{~Hz})$ EBS can reduce interictal epileptiform spikes and seizures, with class-1 evidence from humans supporting HF duty cycle (4) and responsive (1) stimulation. The evidence that LF stimulation can reduce interictal epileptiform spikes and seizures comes from uncontrolled human investigations $(18,46,47)$, non-human primates (48), and rodent models (49-54).

To address the technology gaps in currently available EBS systems we developed and deployed a distributed brain co-processor to investigate patient reported symptoms, automated behavioral state classification, IES biomarkers, and seizures during low and high frequency continuous and adaptive responsive EBS paradigms. Similar to previous studies, we found that patients $(36,55)$, and pet owners do not create reliable seizure diaries when compared to gold-standard seizure catalogs created from automated seizure detection applied to continuous iEEG. This is not surprising given that seizures can be subtle, can go unnoticed by caregivers, and patients are often amnestic for their seizures. This result highlights the challenge of optimizing therapy if the most critical measure of outcome, seizure rates, are inaccurate and this may play a role in the long time required for therapy optimization with current FDA approved devices.

We show that hippocampal IES rates and characteristics are dynamically changing, with an increase in IES during slow-wave sleep in human. Interestingly, seizures exclusively occurred during wakefulness in the human subject despite increased IES rates in slow-wave sleep. Similar to previous reports we did not identify seizures arising from REM sleep $(56,57)$. The EBS paradigms investigated had a modest impact on seizure rates, but the therapeutic benefit may be obscured by the well known implant lesion effect $(1,4)$. It is challenging to recruit for studies during which there is a long post-implant baseline period without EBS given the established benefit of EBS and currently FDA approved devices. Patients and their physicians may appropriately be reluctant to enroll in trials with a 3 to 4 month phase without EBS needed to washout the implant effect $(3,4)$. 
We demonstrated the ability to track behavioral state and show that EBS modifies total sleep time, REM and N2 sleep. Sleep disruption is a common comorbidity of drug resistant epilepsy (58) and EBS therapy can further negatively impact sleep (44). We used behavioral state tracking to investigate a circadian adaptive EBS protocol designed to minimize sleep disruption and deliver increased EBS during periods of increased IES activity and seizures that was better tolerated by patient report than continuous HF stimulation (Table 6). In addition to sleep modulation with EBS therapy, there is emerging evidence that slow-wave sleep may play a role in consolidating seizure engrams and related to epilepsy progression $(59,60)$. Thus, the ability to track wake and sleep behavioral states and adaptively adjust EBS therapy may prove useful for disrupting sleep related seizure consolidation, as well as other future applications.

The current study has a number of limitations. Given the fact that seizures are relatively rare events the accumulation of adequate statistics remains a fundamental challenge for epilepsy research. This was partially mitigated here by quantifying the effect of EBS on IES, a biomarker of pathological brain excitability. Perhaps the biggest limitation is the use of data immediately after implant for baseline seizure and IES rates. There is a well-established lesion effect of ANT and Hc implant on seizure counts that appears to normalize after $3-4$ months based on the Neuropace RNS and Medtronic SANTE trials $(1,4)$. In fact, using only the patient reported diaries the seizure frequency prior to implant was higher (2.6 per week) than post implant (1.4 per week) and during 1 month baseline without the EBS. To investigate efficacy of EBS we used a sequential approach of therapy titration spanning weeks of observation to capture and compare natural circadian and multi-day cycles of IES and seizures $(39,41)$. We started with the lowest $R C+S^{T M}$ stimulation frequency $(2 \mathrm{~Hz})$ possible and sequentially moved to $7 \mathrm{~Hz}$ and $145 \mathrm{~Hz}$. We did not, however, have a period without stimulation for washout between any EBS parameter changes.

In summary, we present results from a powerful system integrating a new investigational neural sensing and stimulation device with local and distributed computing that should prove useful for investigation of EBS in drug resistant epilepsy. The current system places a burden on the patient requiring management and charging of three devices (Fig. 1.; implantable device, CTM, and tablet computer). This research identifies areas for future improvement, including the need for improving bi-directional interfaces to reduce complexity, data loss, and battery life. Future implantable systems with greater device computational power and data storage capacity will enable smart sampling paradigms to buffer data, run embedded algorithms, and trigger alarms for therapy change, behavioral queries, and data transfer that should enhance understanding of behavior and brain activity, and reduce patient burden.

\section{MATERIALS AND METHODS}

\section{Study design and data sources}

To develop classification algorithms, we used a large database of iEEG from two different implanted devices, the NeuroVista and Medtronic devices that wirelessly stream iEEG data. The dataset included 10 humans (NV1-9; MH1) and 11 canines. The dogs MD1-3 were control dogs without epilepsy implanted at Mayo for developing the behavioral state classifiers. The MD4 dog (implanted at Mayo), UCD1 and UCD2 (implanted at UC-Davis), and 5 NeuroVista - dogs with epilepsy implanted across multiple institutions in US $(35,37)$ had naturally occurring epilepsy. We used 10 humans and 8 dogs (MD4, UCD1, UCD2, and 5 NV dogs) for training, validation, and pseudo-prospective testing seizure and IES detection algorithms. An automated algorithm for brain state classification was developed using simultaneous polysomnograms and iEEG collected from 3 canines (MD1-3) and a 
human subject $(\mathrm{MH} 1)$ implanted with the investigational Medtronic Summit $R C+S^{T M}$ device $\left(R C+S^{T M}\right)$. Then classification algorithms were prospectively tested within a distributed brain co-processor for neurophysiologic tracking and adaptive stimulation in 2 pet dogs (UCD1, UCD2) and the human subject (MH1) in their natural environments.

\section{Devices, training, validation, and testing data}

Datasets collected from two implantable devices were utilized for system training, validation, and testing (Table 1). The investigational NeuroVista system is a 16 -channel brain sensing $(0.1-100 \mathrm{~Hz}$ bandwidth; $400 \mathrm{~Hz}$ sampling) implantable device providing continuous iEEG wireless streaming to an off-the-body data storage and analytics device carried by the patients and dogs. The RC+S ${ }^{\mathrm{TM}}$ is a 16 channel electrical stimulation and sensing implantable device capable of selective sensing from any 4 of the 16 channels $(1-70,125,250 \mathrm{~Hz}$ bandwidth; programmable sampling 250, 500, or 1000 $\mathrm{Hz}$ ) and wireless streaming to a handheld tablet computer with cellular and internet connectivity to a central cloud based data and analytics platform $(22,23)$. The investigational NeuroVista and $R C+S^{T M}$ devices have yielded massive datasets of ambulatory iEEG in naturalistic settings and are idea for development of robust automated algorithms for brain behavioral state classification, IES and seizure detection. We have previously used the NeuroVista Inc. device data from humans (36) and canines (34) for developing seizure detection and forecasting algorithms $(35,61-63)$.

Canine Device Implants: The animal research and clinical care took place at Mayo Clinic, Rochester MN and University of California Davis, Davis, CA under IACUC Protocol A00002655 Chronic Wireless Electrophysiology and Modulation in Epileptic Dogs. Epilepsy occurs naturally in dogs with prevalence, age of onset, and clinical presentation similar to human epilepsy (64). Naturally occurring canine epilepsy is often drug resistant and new therapies are needed. In addition, the canines provide a platform for preclinical testing, since dogs are large enough to accommodate devices designed for humans. All canines were implanted at either Mayo Clinic (MD 1-4) or at University of California, Davis (UCD1-2).

Electrode and $\mathrm{RC}+\mathrm{S}^{\mathrm{TM}}$ implantation in dogs: Medtronic deep brain stimulation electrodes were implanted intracranially in canines under anesthesia using a custom made stereotactic frame. Canines underwent a 3.0T MRI using a stereotactic T1-weighted sequence (Fig. 6). Targets and trajectories were planned using stereotactic software (Compass ${ }^{\mathrm{TM}}$ Stereotactic Systems) adapted for a large animal head frame. Burr holes were drilled into the skull for each of the four electrodes (Medtronic models 3391 and 3387) that were inserted to the target depth and secured with metal anchors and bone screws. The electrode tails were tunneled to the $R C+S^{T M}$ in a pocket behind the canines right scapula. The canine underwent a post-op x-ray CT scan, which was then co-registered to the stereotactic MRI (Analyze 12.0, BIR, Mayo Foundation) in order to verify targeting accuracy. We have previously described the similar procedure for the previous NeuroVista Inc. device implants carried out at Mayo Clinic, University of Minnesota, University of Pennsylvania, and University of California Davis in canines $(34,35)$. 


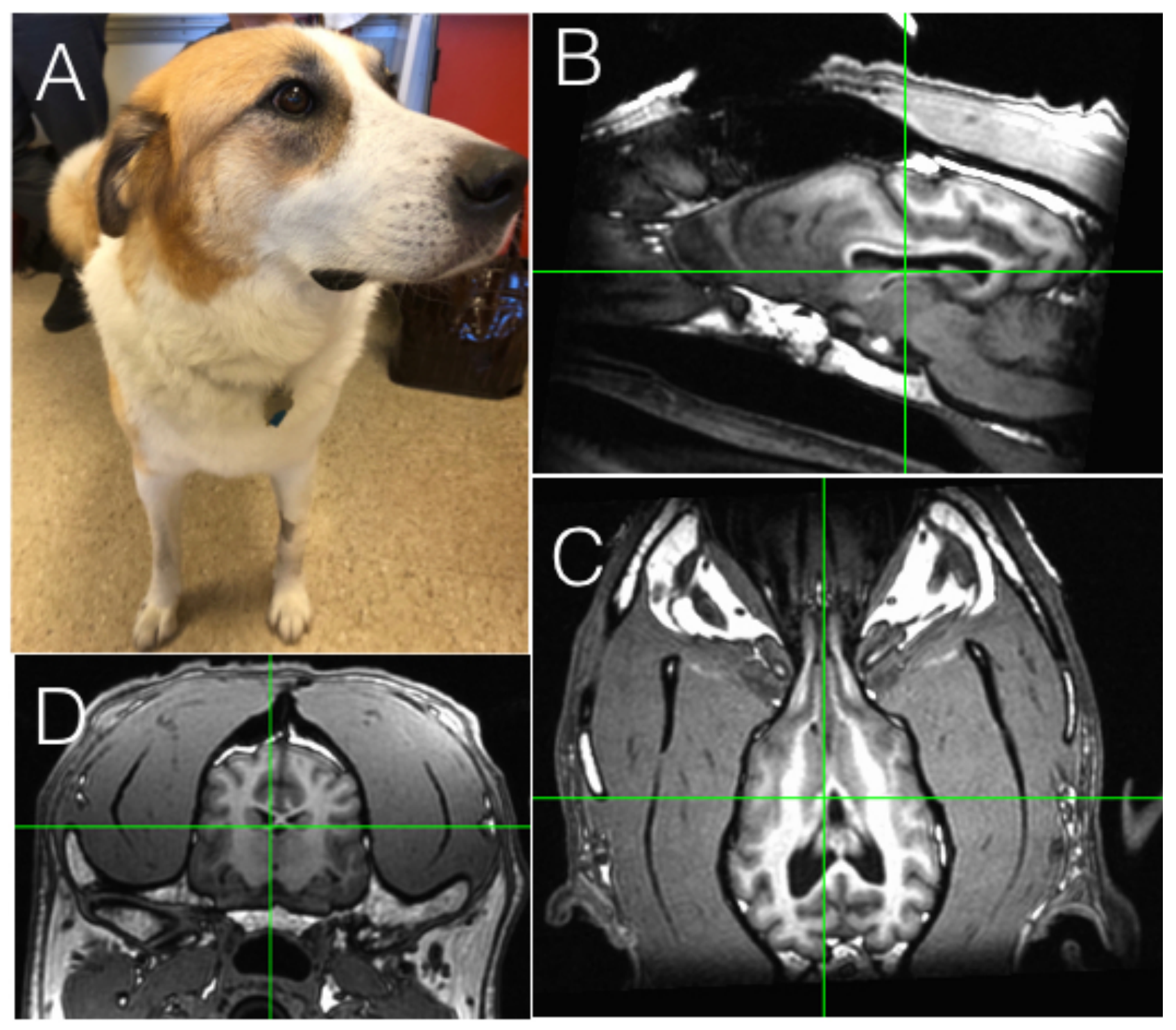

Fig. 6. Canine stereotactic implant. (A) 6 yr. old pet dog with drug resistant epilepsy. High resolution (B) Sagittal. (C) Axial. (D) Coronal T1 MRI. The electrode implants are by direct visual targeting of anterior nucleus of thalamus and hippocampus.

Human implant: The human subject research was carried out under an FDA IDE: G180224 and Mayo Clinic IRB: 18-005483 "Human Safety and Feasibility Study of Neurophysiologically Based Brain State Tracking and Modulation in Focal Epilepsy". The study is registered at https://clinicaltrials.gov/ct2/show/NCT03946618. The patients provided written consent in accordance with the IRB and FDA requirements.

Subject $\mathrm{MH} 1$ is a 57 -year-old ambidextrous woman with drug resistant temporal lobe epilepsy following head trauma in childhood. She suffered her first generalized tonic-clonic seizure after a head injury at age 9 . Her seizures were well-controlled by an anti-seizure medication regimen until college (at 21 yrs.), when her seizures became drug resistant. At time of device implant, she had suffered from more than 30 years of drug resistant epilepsy, depression and anxiety. Electrode and $R C+S^{\mathrm{TM}}$ implantation in human: The details of the approach for implantation have been previously described (65). Magnetic resonance imaging (MRI) was performed after Leksell (Elekta Inc.) frame fixation for 
stereotactic targeting. Medtronic 3387s electrodes were then implanted in the ANT by direct targeting of the mammillothalamic tract on MRI (FGATIR sequence (66)). Medtronic 3391 electrodes were implanted into the hippocampus through direct targeting of the amygdala and hippocampal head. After confirmation of the electrode location with intraoperative computed tomography (CT), the leads were connected to bifurcated extensions and tunneled to the $R C+S^{T M}$ in an infraclavicular pocket (Fig. 7). The 10 human dataset collected with the NeuroVista device were from the NeuroVista Inc. device trial in humans carried out in Melbourne, Australia, between March 24, 2010, and June 21, 2011 (36).

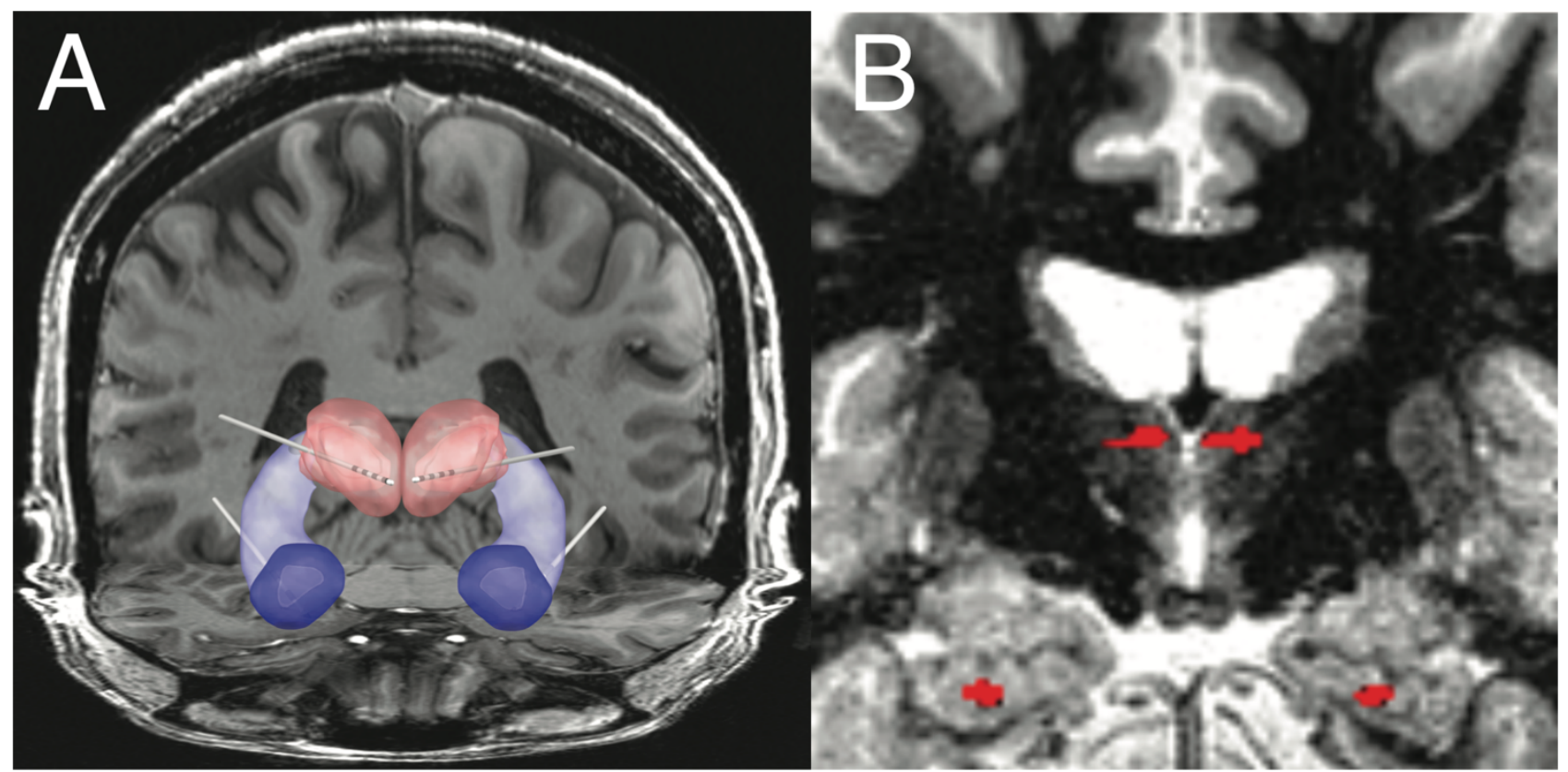

Fig. 7. Human subject MH1. Bilateral Anterior Nucleus Thalamus (ANT) and Hippocampus (HC) implant. (A) Papez circuit and implanted electrodes. (B) MRI - the ANT and HC electrodes from co-registration of MRI and post-implant CT are highlighted in red.

\section{Automated Sleep Classification}

We trained and deployed the automated sleep classification on datasets recorded with $R C+S^{T M}$ system (23). In order to get reliable sleep classification for longterm iEEG data, we trained a subject specific sleep classifier for each subject (human subject, MH1 and three dogs MD1, 2, 3) using a single channel of iEEG data with gold standard sleep annotations obtained from simultaneously recorded polysomnograms. In the human study we recorded three nights under different EBS conditions (ANT stimulation: off, frequency $2 \mathrm{~Hz}, 7 \mathrm{~Hz}, 100 \mathrm{~Hz}$, current $3 \mathrm{~mA}$, pulse width $90 \mu \mathrm{sec}$ ) with simultaneous scalp polysomnography (PSG) recording and continuous iEEG data streaming (both ANT and Hc electrodes) from the implanted $\mathrm{RC}+\mathrm{S}^{\mathrm{TM}}$ as shown in Fig. 8. The PSG signals were scored by an expert into standard sleep categories (Wake, Rapid-Eye-Movement (REM), and three non-REM stages (N1, N2, N3) using AASM 2012 scoring rules (29). In three 
dogs, the iEEG data were recorded from various brain locations to determine the classifier performance in different brain targets (pre-frontal cortex, ANT, Hc), while simultaneously recording scalp EEG, accelerometry data and video recordings. The scalp EEG, accelerometry and video recording were used to assess the sleep stage by expert manual review. In all three dogs, different continuous EBS stimulation conditions (off, frequency $2 \mathrm{~Hz}, 7 \mathrm{~Hz}$, amplitude $3 \mathrm{~mA}, 200 \mathrm{us}$ pulse width) were studied in subsequent nights to compare classifier performance under different stimulation settings with ongoing ANT stimulation. The classifier performance was measured as a harmonic mean of the precision and recall (F1-score).

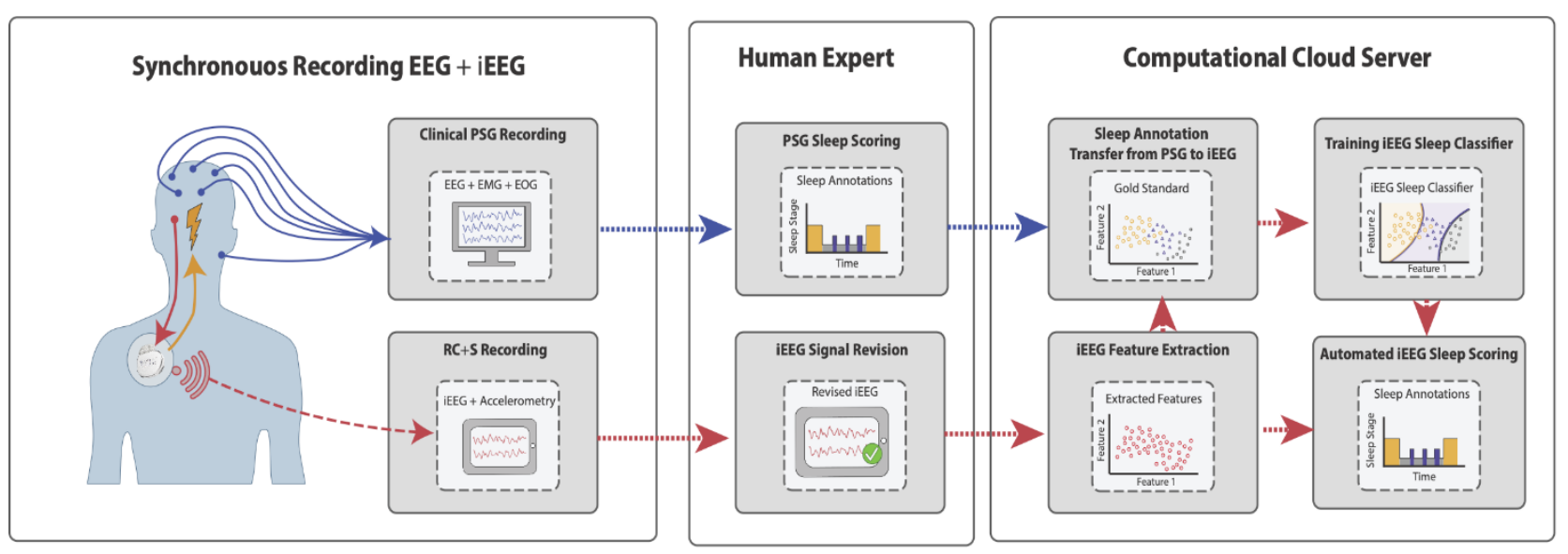

Fig. 8. Block-diagram of automated sleep classification trials for human study. First, the EEG and iEEG data are acquired using standard polysomnogram (PSG) clinical beside system and experimental Medtronic Summit RC+S implantable system. The data are synchronized using annotations and electrical stimulation artifacts. An expert then scores the PSG data to create a gold-standard sleep scoring dataset. Human operator also has the option to review the iEEG for data drops and artifacts. Training and deploying of automated sleep classifier are performed in subsequent steps.

We trained a subject specific sleep classifier (Naïve Bayes) for following subjects: one human patient and three dogs. The classifier was trained using the first night of data, which were always without stimulation. The second night was used for validation and the third night for pseudo-prospective testing. The Naïve Bayes classifier uses features extracted from the iEEG data that were previously determined to be useful for behavioral state classification $(25,67)$. The following frequency domain features for each of the 30-second iEEG epoch were used: mean dominant frequency, absolute and relative mean band power for frequency bands: Delta $(1-4 \mathrm{~Hz})$, Theta $(4-8 \mathrm{~Hz})$, Alpha $(8-12 \mathrm{~Hz})$ and four high frequency bands, Beta bands $(12-16 \mathrm{~Hz} ; 16-20 \mathrm{~Hz} ; 20-30 \mathrm{~Hz})$ and gamma band $(30-40 \mathrm{~Hz})$. We also recombined absolute band power feature values to obtain ratios such as Theta-to-Beta ratio, etc., for all ascending frequency bands. Subsequently, we performed preprocessing feature selection and dimensionality reduction using Principal Component Analysis (PCA) followed by an additional feature selection (Fig. 9.). To prepare the training data, we filtered the outliers by categorywise outlier filtering. As a post-processing step, after the class-probability prediction, the class-probabilities were corrected using Bayes Theorem and state-to-state transition probabilities inferred from publicly available PSG dataset (68). The framework we implemented and used for this classifier is publicly available: https://github.com/mselair/AISC 


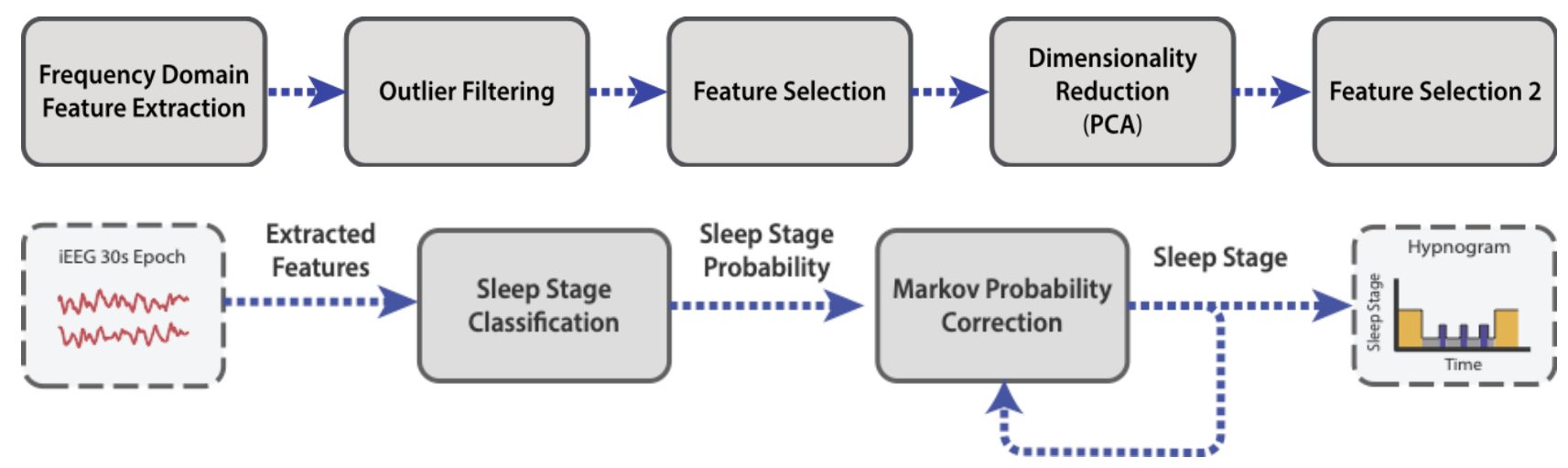

Fig. 9. Sleep Classification pipeline. Feature pre-processing is used to clean the features for training the classifier. Postprocessing using a statistical inference (Bayesian inference) on predicted classification score correction to correct possible classification errors.

\section{Detection of interictal epileptiform spikes}

Interictal epileptiform spikes are an electrographic marker of pathologic brain tissue capable of generating unprovoked seizures. In recent years there has been rapid development of new and reliable techniques for automated IES detection. To train and evaluate the IES detector we used continuous hippocampal recordings from the $R C+S^{T M}$ (23). We used a previously validated algorithm (42) that models and adapts based on statistical distributions of signal envelopes from background (normal) iEEG activity. This enables differentiating signals containing IESs from signals with background activity even in long term data recordings with changing background electrophysiological activity. The IES detector also identified low-amplitude IES in cases where the background activity power is low and IES are often missed by expert visual review.

We benchmarked the IES detector using data acquired with a chronically implanted brain stimulator $\left(R C+S^{T M}\right)$. We deployed the detector in a cloud system that received the continuously streaming hippocampal data over one year. We compared the detector performance with the manual visual review (GW \& NG electroencephalographers) scoring in selected epochs (see Data for IES Detector). The IES detector ran during different stimulation paradigms, no stimulation, 2, 7, and $145 \mathrm{~Hz}$ stimulation) with changing stimulation current amplitudes (2, 3, $5 \mathrm{~mA})$ and pulse widths of 90 and 200 usec.

To investigate how IESs characteristics change in periods of different seizure frequency we selected epochs of the data in periods of frequent (cluster) and less frequent seizure activity (non-cluster). The seizure cluster period was defined as more than two seizures in a day. For each of the two (cluster, non-cluster) we selected 5-minute-long epochs for left and right hippocampal channels. Each selected epoch was taken at distinct times to assess differences between sleep and wake cycles. In total we selected twenty-four 5-minutes long epochs reviewed independently by two electroencephalographers. All IESs were marked in both hippocampal channels and used subsequently to calculate congruence score between experts and to validate the automated IES detector. Subsequently, we used the two-months period of the data continuously streamed from the human with implanted $R C+S^{T M}$ to analyze IES rates and IES characteristics. 


\section{Generic seizure detector}

The training dataset consists of long term NeuroVista recordings from 5 canines and 2 human patients (Table 2). In canines, all seizures were included in training (340 in total). Another 628 interictal segments with various electrophysiological activity patterns were manually selected. The human dataset consists of 1049 seizures and 846 interictal segments. Half of the seizures (524) and half of the interictal segments (423) were bootstrapped and used as training data and the other half of data used in the validation dataset. The validation dataset included two sets of data. The first dataset has data from $\mathrm{RC}+\mathrm{S}^{\mathrm{TM}}$ recordings from three canines. Each recording spans at least 210 days. In total, 133 electrographic seizures and 833 interictal segments were selected from the continuous recordings upon visual review by an expert reviewer. The second dataset contains the other half of the data (2 NeuroVista patient recordings) generated by bootstrapping in the training dataset.

The testing datasets include previously collected NV dataset that were used for pseudo-prospective testing and $\mathrm{RC}+\mathrm{S}^{\mathrm{TM}}$ datasets for prospective testing. The pseudo-prospective NeuroVista human data was from 7 patients and true prospective deployment of the model in one human patient and two pet canines implanted with the $R C+S^{T M}$ system. The pseudo-prospective NV human dataset period is $\sim 10.5$ years and includes 2046 seizures in total. The prospective deployment ran over 374 days and contains data of 134 seizures that were recorded in the human $R C+S^{\mathrm{TM}}$ system and 23 seizures recorded in the two pet canines (UCD1 and UCD2).

\section{Detector Design - utilizing LSTM neural network}

To design a generalizable seizure detection algorithm for a generic implantable system, we required the algorithm operate independently of the recording system, spatial electrode position, and species tested. We used two of the few implantable neuro-devices capable of continuous streaming local field potential data through a wireless connection. This allows long-term, real-time monitoring since the collected data are continually transferred from the implantable device to the brain co-processer system (tablet or smartphone, and cloud computational resource) (23). For this reason, the algorithm must be capable of processing data streams with artifacts and data drops caused by interference or disconnections.

Seizures recorded with intracranially implanted electrodes exhibit temporal evolution of spectral power across a wide range of frequencies. Different electrographic signatures are observed in the data based on their initial power distribution. It is important to note that seizures in one patient might have multiple ictal patterns, therefore training on different ictal patterns is necessary for high sensitivity seizure detection. The detector has to distinguish ictal patterns from sharp transient artifacts coming from a recording device or short interictal discharges which might temporarily increase spectral power similar to an electrographic seizure.

Previously reported seizure detectors $(62,69-71)$ usually utilize combination of features extracted from multiple channels, or features extracted from shorter segments without adaptation to a longterm baseline. This is a crucial design input requirement in designing the seizure detector for a longterm monitoring in chronically implanted devices. Another drawback of previously reported detectors is that the testing is usually done on isolated ictal and interictal segments, and not on a long-term continuous recording spanning weeks and months of time. The deployment of trained and validated seizure detectors on previously unseen out of sample unbalanced data is critical for evaluation and real-time performance of a generalizable seizure detector. Our method focuses on spectral changes in iEEG recordings from only one channel and marks a probability of seizure over time thus providing 
independence from the neighboring channels and the short interictal discharges that could confuse current state of the art detectors.

In order to address these requirements, we developed a convolutional long short-term memory (LSTM) $(32,33)$ neural network utilizing Short Time Fourier Transform (STFT) calculated from single lead iEEG as an input. We previously used CNN with LSTM for automated classification of iEEG (72). The STFT converts the single lead time series data into time-frequency representation (spectrogram). Invariance to sampling frequency is achieved by using a constant time window of 1 second with 0.5 seconds overlap, and subsequently selecting only frequencies lower than $100 \mathrm{~Hz}$. A raw data segment is always transformed into a spectrogram image with dimensions $100 x \mathrm{~T}$, where $\mathrm{T}$ is the number FFT calculations, not depending on sampling frequency (frequency domain resolution is always $1 \mathrm{~Hz}$ per sample). Time series data of $5 \mathrm{~min}$ length were empirically chosen to provide long enough EEG baseline temporal context for the LSTM, so the relative power of seizure stands out of the background activity. The final classification is made for every $0.5 \mathrm{sec}$ of the 5 -minute input raw data signal using a many-to-many LSTM architecture. Raw data are z-score normalized prior to STFT calculation and each frequency band of the resulting spectrogram is z-score normalized prior to the neural network inference. Dropout layers in neural networks are used for regularization during training to prevent overfitting. Similarly, we drop random segments prior to the spectrogram computation. This enables the network to handle the data from the wireless system with possible short data gaps.

The convolutional LSTM model consists of 2 convolutional blocks (convolution and ReLU) with kernels $\{5,5\}$ and $\{96,3\}$, respectively. Subsequently, time distributed feature representation is processed with 2 layers of bidirectional LSTM recurrent neural network. Lastly, a fully connected layer with a softmax activation function transforms the LSTM output into probability output. The proposed architecture is trained with Adam optimizer (learning rate $=10^{-3}$, weight regularization $=10^{-4}$ ) in a many-to-many training scheme, where every input FFT window has a multiclass label. We implemented 4 types of labels - normal activity, IES together with artifacts, dropout segments, and seizures. Adding additional labels might improve learning because the model is forced to not only distinguish interictal activity from continuous seizure activity but also interictal discharges which are not considered as electrographic seizures in different behavioral states, and thus lower the number of false positives. The temporal resolution of the detector is defined by the FFT window step ( 0.5 seconds). In order to train the network, we use a special purpose deep learning computer Lambda Labs Inc. (8x GTX 2080TI GPU, 64 CPU cores and 512 GB RAM). The data-parallel training method runs on all GPUs and average model gradients and is used to reduce training time. The model is built in the PyTorch deep-learning library for Python.

\section{Training and validation of seizure detection model}

The model was trained on NeuroVista data ( 5 canines, 2 human patients, Table 2 ). All training segments were 10 minutes long. Random 5 minutes intervals were sampled from the full segments during the training every time the segment was used in training. Because the human training dataset had a higher number of examples than the canine training dataset during the training epoch training examples were randomly sampled in a way that the number of examples from both classes was balanced.

Performance of the model during the training was evaluated by area under the precision-recall curve (AUPRC), where all seizure targets were set to 1 and all the other classes were set to 0 . Validation 
of CNNs is typically measured by validation loss, but we used AUPRC for scoring because it is independent of the probability threshold of the classifier and it is not dependent on the true negative samples in the dataset. Validation examples were fixed 5 minute intervals and were not randomly sampled. Validation scores (AUPRC) were calculated on two different datasets (3 canines with $R C+S^{T M}, 2$ human patients with NeuroVista device) independently. The two validation scores were averaged after each training epoch and the model with the best score achieved during training was deployed on the test dataset in order to obtain results (Table 2).

\section{Model deployment}

We arbitrarily chose 10 continuous seconds of ictal activity as an electrographic event that we want to detect (73). The model iterates over the data with 5-minute windows with 100 seconds of overlap. The model gives a probability of seizure for every 0.5 seconds (higher probability is used in the overlap region) in every channel. Seizures in the test dataset are marked across all channels without specification therefore we combine probabilities from all channels in the following way. The three highest probabilities from all channels are averaged and from this averaged probability the final performance measures are calculated. For a given probability threshold the system identified continuous detection whenever the probability was above a threshold (see example of a detection in Fig. 3 and 10). Next, every detection interval above a threshold was automatically extended if in the next 10 seconds from the current detection was another detection. Subsequently, the two detections were merged into one interval. Thus, for every probability threshold, we detected intervals of various lengths which the model marks as seizures. Intervals shorter than 10 seconds were dropped from detected events. For detected events longer than 10 seconds AUPRC and AUROC scores were calculated based on the region overlap with gold standard seizures marked by an expert reviewer.

The model was deployed in local cloud storage to continuously process incoming data from $R C+S^{\mathrm{TM}}$ animal and human study. Due to a different electrode configuration in the $R C+S^{T M}$ system in comparison with the NeuroVista system, we could not use an average of the three highest probabilities. Instead, a maximal probability given by two hippocampal channels was taken as an output of the model. Subsequently, the detected intervals were calculated from the probabilities in the same manner as for the data from the NeuroVista dataset. The model has been running online and continuously detecting seizure events as the new data were coming in. A revision of the raw data by an expert reviewer created gold standard seizure marks for comparison of classifier performance. Thus, with all detected events and true seizure marks AUPRC and AUROC scores were calculated.

The performance of the model on out-of-sample data is numerically shown in Table 3 . The performance of the generalized classifier is visualized using standard machine learning graphs of Precision Recall (PRC) and Receiver Operating Characteristic (ROC) curves for each individual human (Fig. 4 ). The results of model detections outperform state of the art detectors published recently Baldassano, Brinkmann et. al (62) and directly compare two hundred teams of data scientists across the globe comprising 241 individuals. An advantage over a Kaggle competition we were able to take and use a larger portion of the full dataset in a more realistic setting, where the classifier is trained and then pseudo-prospectively run on the new out-of-sample data of different subjects in a sequential way fully simulating a real prospective situation of recording where new data are arriving each second and detector runs in near real-time manner. Yet, the classifier doesn't need to be retrained for patient specific applications and is fully generalized. On the other hand, Fig. 10 shows an example of a short period (a minute) of iEEG data with seizure for all sixteen neocortical electrodes of patient N7 from NV human dataset. The seizure is visually apparent in only a few channels with adequate 
signal to background ratio suitable for automated detections. This is likely a common situation with electrodes spanning the space from seizure onset zone to surrounding regions of the brain. Fig. 10 reveals the time-frequency analysis of these iEEG signals showing the different signatures of seizure electrophysiology in different channels and below is the visualization of the classifier output probabilities for each electrode. This also shows in the time-frequency domain that for some electrodes the seizure is very prominent while for others not differentiable from the background signal. Therefore, here the model decides based on the seizure probabilities of the electrodes taken as a mean of top three probabilities.

A

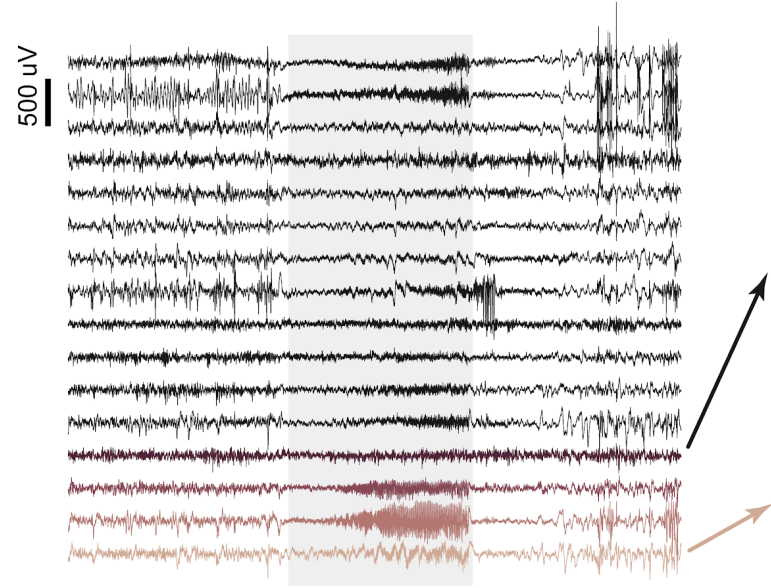

C

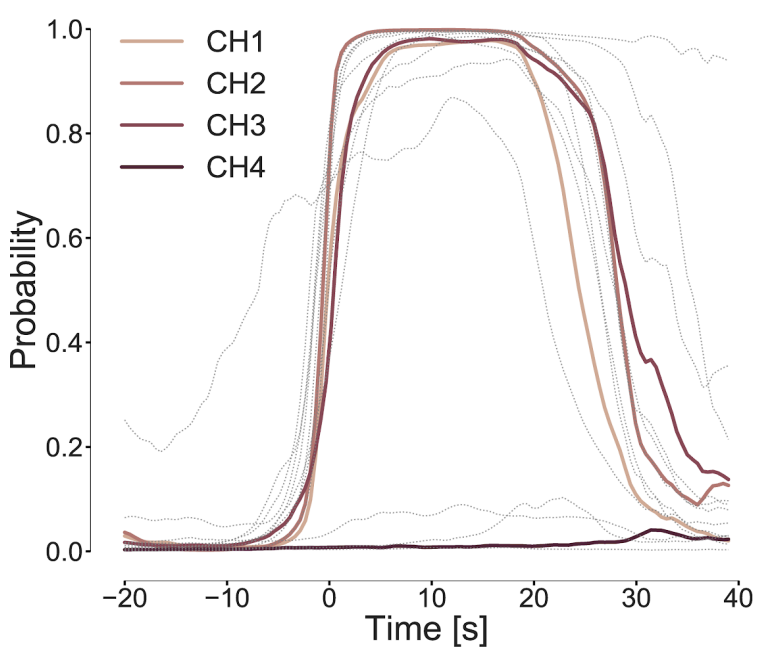

B

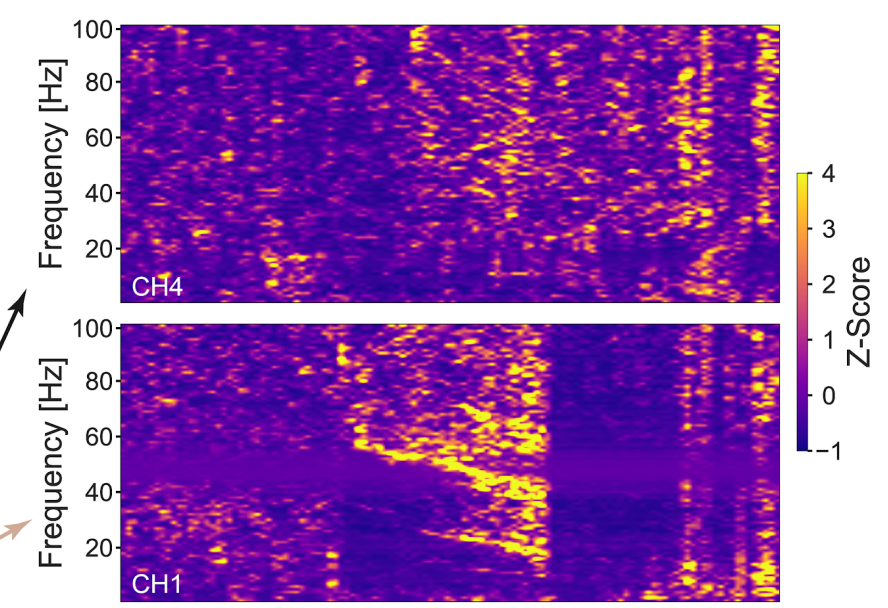

$\mathrm{D}$

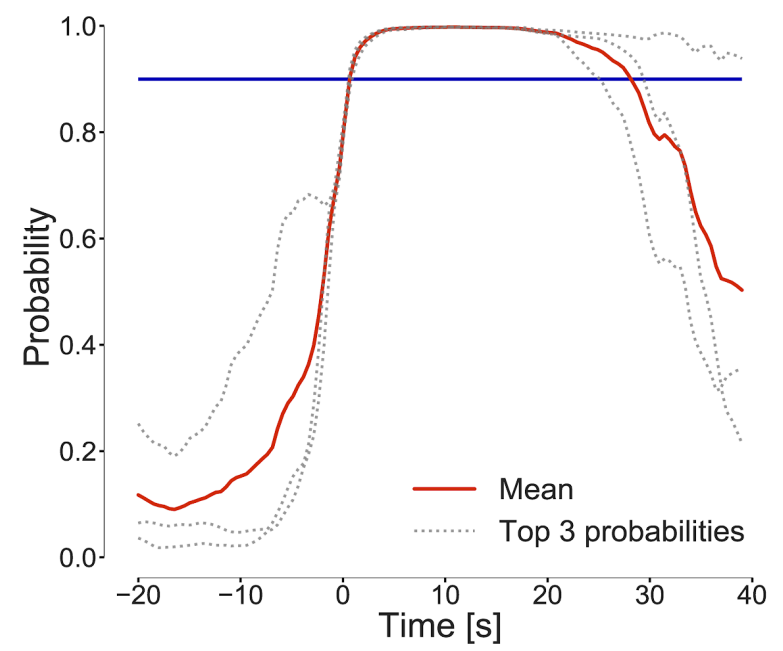

Fig. 10. Spectral features of spontaneous seizures. (A) One minute of iEEG data recorded with NV device, sixteen neocortical electrodes, containing a spontaneous human seizure. The seizure is present on a few channels with a good signal to background ratio suitable for automated detection. (B) Time-frequency analysis of signals show the different signatures of seizure electrophysiology (shaded area) in different channels: channel 1, where seizure is notable and channel number 4 where it is hard to identify the seizure. (C) Plots of classifier probabilities for each electrode below actual raw data showing that for some electrodes the seizure is very prominent and for some not differentiable from the background signal. (D) The classifier output probabilities for top three probabilities together with the mean (red) and threshold (blue) showing when the detection is raised (time $0)$. 


\section{References:}

1. M. J. Morrell, Responsive cortical stimulation for the treatment of medically intractable partial epilepsy, Neurology 77, 1295-1304 (2011).

2. G. K. Bergey, M. J. Morrell, E. M. Mizrahi, A. Goldman, D. King-Stephens, D. Nair, S. Srinivasan, B. Jobst, R. E. Gross, D. C. Shields, G. Barkley, V. Salanova, P. Olejniczak, A. Cole, S. S. Cash, K. Noe, R. Wharen, G. Worrell, A. M. Murro, J. Edwards, M. Duchowny, D. Spencer, M. Smith, E. Geller, R. Gwinn, C. Skidmore, S. Eisenschenk, M. Berg, C. Heck, P. Van Ness, N. Fountain, P. Rutecki, A. Massey, C. O'Donovan, D. Labar, R. B. Duckrow, L. J. Hirsch, T. Courtney, F. T. Sun, C. G. Seale, Long-term treatment with responsive brain stimulation in adults with refractory partial seizures, Neurology 84, 810817 (2015).

3. D. R. Nair, K. D. Laxer, P. B. Weber, A. M. Murro, Y. D. Park, G. L. Barkley, B. J. Smith, R. P. Gwinn, M. J. Doherty, K. H. Noe, R. S. Zimmerman, G. K. Bergey, W. S. Anderson, C. Heck, C. Y. Liu, R. W. Lee, T. Sadler, R. B. Duckrow, L. J. Hirsch, R. E. Wharen, W. Tatum, S. Srinivasan, G. M. McKhann, M. A. Agostini, A. V. Alexopoulos, B. C. Jobst, D. W. Roberts, V. Salanova, T. C. Witt, S. S. Cash, A. J. Cole, G. A. Worrell, B. N. Lundstrom, J. C. Edwards, J. J. Halford, D. C. Spencer, L. Ernst, C. T. Skidmore, M. R. Sperling, I. Miller, E. B. Geller, M. J. Berg, A. J. Fessler, P. Rutecki, A. M. Goldman, E. M. Mizrahi, R. E. Gross, D. C. Shields, T. H. Schwartz, D. R. Labar, N. B. Fountain, W. J. Elias, P. W. Olejniczak, N. R. Villemarette-Pittman, S. Eisenschenk, S. N. Roper, J. G. Boggs, T. A. Courtney, F. T. Sun, C. G. Seale, K. L. Miller, T. L. Skarpaas, M. J. Morrell, Nine-year prospective efficacy and safety of brain-responsive neurostimulation for focal epilepsy, Neurology 95, e1244-e1256 (2020).

4. R. Fisher, V. Salanova, T. Witt, R. Worth, T. Henry, R. Gross, K. Oommen, I. Osorio, J. Nazzaro, D. Labar, M. Kaplitt, M. Sperling, E. Sandok, J. Neal, A. Handforth, J. Stern, A. DeSalles, S. Chung, A. Shetter, D. Bergen, R. Bakay, J. Henderson, J. French, G. Baltuch, W. Rosenfeld, A. Youkilis, W. Marks, P. Garcia, N. Barbaro, N. Fountain, C. Bazil, R. Goodman, G. McKhann, K. Babu Krishnamurthy, S. Papavassiliou, C. Epstein, J. Pollard, L. Tonder, J. Grebin, R. Coffey, N. Graves, Electrical stimulation of the anterior nucleus of thalamus for treatment of refractory epilepsy, Epilepsia 51, 899-908 (2010).

5. V. Salanova, T. Witt, R. Worth, T. R. Henry, R. E. Gross, J. M. Nazzaro, D. Labar, M. R. Sperling, A. Sharan, E. Sandok, A. Handforth, J. M. Stern, S. Chung, J. M. Henderson, J. French, G. Baltuch, W. E. Rosenfeld, P. Garcia, N. M. Barbaro, N. B. Fountain, W. J. Elias, R. R. Goodman, J. R. Pollard, A. I. Troster, C. P. Irwin, K. Lambrecht, N. Graves, R. Fisher, Long-term efficacy and safety of thalamic stimulation for drug-resistant partial epilepsy, Neurology 84, 1017-1025 (2015).

6. A. Kupsch, R. Benecke, J. Müller, T. Trottenberg, G.-H. Schneider, W. Poewe, W. Eisner, A. Wolters, J.-U. Müller, G. Deuschl, M. O. Pinsker, I. M. Skogseid, G. K. Roeste, J. Vollmer-Haase, A. Brentrup, M. Krause, V. Tronnier, A. Schnitzler, J. Voges, G. Nikkhah, J. Vesper, M. Naumann, J. Volkmann, Pallidal Deep-Brain Stimulation in Primary Generalized or Segmental Dystonia, N. Engl. J. Med. 355, 1978-1990 (2006).

7. G. Deuschl, C. Schade-Brittinger, P. Krack, J. Volkmann, H. Schäfer, K. Bötzel, C. Daniels, A. Deutschländer, U. Dillmann, W. Eisner, D. Gruber, W. Hamel, J. Herzog, R. Hilker, S. Klebe, M. Kloß, J. Koy, M. Krause, A. Kupsch, D. Lorenz, S. Lorenzl, H. M. Mehdorn, J. R. Moringlane, W. Oertel, M. O. Pinsker, H. Reichmann, A. Reuß, G.-H. Schneider, A. Schnitzler, U. Steude, V. Sturm, L. Timmermann, V. Tronnier, T. Trottenberg, L. Wojtecki, E. Wolf, W. Poewe, J. Voges, A Randomized Trial of Deep-Brain Stimulation for Parkinson's Disease, N. Engl. J. Med. 355, 896-908 (2006). 
8. R. F. Dallapiazza, D. J. Lee, P. De Vloo, A. Fomenko, C. Hamani, M. Hodaie, S. K. Kalia, A. Fasano, A. M. Lozano, Outcomes from stereotactic surgery for essential tremor, J. Neurol. Neurosurg. Psychiatry 90, 474-482 (2019).

9. B. D. Greenberg, L. A. Gabriels, D. A. Malone, A. R. Rezai, G. M. Friehs, M. S. Okun, N. A. Shapira, K. D. Foote, P. R. Cosyns, C. S. Kubu, P. F. Malloy, S. P. Salloway, J. E. Giftakis, M. T. Rise, A. G. Machado, K. B. Baker, P. H. Stypulkowski, W. K. Goodman, S. A. Rasmussen, B. J. Nuttin, Deep brain stimulation of the ventral internal capsule/ventral striatum for obsessive-compulsive disorder: worldwide experience, Mol. Psychiatry 15, 6479 (2010).

10. H. S. Mayberg, A. M. Lozano, V. Voon, H. E. McNeely, D. Seminowicz, C. Hamani, J. M. Schwalb, S. H. Kennedy, Deep Brain Stimulation for Treatment-Resistant Depression, Neuron 45, 651-660 (2005).

11. T. E. Schlaepfer, M. X. Cohen, C. Frick, M. Kosel, D. Brodesser, N. Axmacher, A. Y. Joe, M. Kreft, D. Lenartz, V. Sturm, Deep Brain Stimulation to Reward Circuitry Alleviates Anhedonia in Refractory Major Depression, Neuropsychopharmacology 33, 368-377 (2008).

12. D. A. Malone, D. D. Dougherty, A. R. Rezai, L. L. Carpenter, G. M. Friehs, E. N. Eskandar, S. L. Rauch, S. A. Rasmussen, A. G. Machado, C. S. Kubu, A. R. Tyrka, L. H. Price, P. H. Stypulkowski, J. E. Giftakis, M. T. Rise, P. F. Malloy, S. P. Salloway, B. D. Greenberg, Deep Brain Stimulation of the Ventral Capsule/Ventral Striatum for TreatmentResistant Depression, Biol. Psychiatry 65, 267-275 (2009).

13. M. T. Kucewicz, B. M. Berry, L. R. Miller, F. Khadjevand, Y. Ezzyat, J. M. Stein, V. Kremen, B. H. Brinkmann, P. Wanda, M. R. Sperling, R. Gorniak, K. A. Davis, B. C. Jobst, R. E. Gross, B. Lega, J. Van Gompel, S. M. Stead, D. S. Rizzuto, M. J. Kahana, G. A. Worrell, Evidence for verbal memory enhancement with electrical brain stimulation in the lateral temporal cortex, Brain 141, 971-978 (2018).

14. Y. Ezzyat, P. A. Wanda, D. F. Levy, A. Kadel, A. Aka, I. Pedisich, M. R. Sperling, A. D. Sharan, B. C. Lega, A. Burks, R. E. Gross, C. S. Inman, B. C. Jobst, M. A. Gorenstein, K. A. Davis, G. A. Worrell, M. T. Kucewicz, J. M. Stein, R. Gorniak, S. R. Das, D. S. Rizzuto, M. J. Kahana, Closed-loop stimulation of temporal cortex rescues functional networks and improves memory, Nat. Commun. 9, 365 (2018).

15. A. W. Laxton, D. F. Tang-Wai, M. P. McAndrews, D. Zumsteg, R. Wennberg, R. Keren, J. Wherrett, G. Naglie, C. Hamani, G. S. Smith, A. M. Lozano, A phase I trial of deep brain stimulation of memory circuits in Alzheimer's disease, Ann. Neurol. 68, 521-534 (2010).

16. A. M. Lozano, L. Fosdick, M. M. Chakravarty, J.-M. Leoutsakos, C. Munro, E. Oh, K. E. Drake, C. H. Lyman, P. B. Rosenberg, W. S. Anderson, D. F. Tang-Wai, J. C.

Pendergrass, S. Salloway, W. F. Asaad, F. A. Ponce, A. Burke, M. Sabbagh, D. A. Wolk, G. Baltuch, M. S. Okun, K. D. Foote, M. P. McAndrews, P. Giacobbe, S. D. Targum, C. G. Lyketsos, G. S. Smith, A Phase II Study of Fornix Deep Brain Stimulation in Mild Alzheimer's Disease, J. Alzheimer's Dis. 54, 777-787 (2016).

17. A. M. Lozano, N. Lipsman, H. Bergman, P. Brown, S. Chabardes, J. W. Chang, K. Matthews, C. C. Mclntyre, T. E. Schlaepfer, M. Schulder, Y. Temel, J. Volkmann, J. K. Krauss, Deep brain stimulation: current challenges and future directions, Nat. Rev. Neurol. 15, 148-160 (2019).

18. B. N. Lundstrom, J. Van Gompel, F. Khadjevand, G. Worrell, M. Stead, Chronic subthreshold cortical stimulation and stimulation-related EEG biomarkers for focal epilepsy, Brain Commun. 1 (2019), doi:10.1093/braincomms/fcz010.

19. H. Cagnan, T. Denison, C. Mclntyre, P. Brown, Emerging technologies for improved 
deep brain stimulation, Nat. Biotechnol. 37, 1024-1033 (2019).

20. A. Ramirez-Zamora, J. Giordano, A. Gunduz, J. Alcantara, J. N. Cagle, S. Cernera, P. Difuntorum, R. S. Eisinger, J. Gomez, S. Long, B. Parks, J. K. Wong, S. Chiu, B. Patel, W. M. Grill, H. C. Walker, S. J. Little, R. Gilron, G. Tinkhauser, W. Thevathasan, N. C. Sinclair, A. M. Lozano, T. Foltynie, A. Fasano, S. A. Sheth, K. Scangos, T. D. Sanger, J. Miller, A. C. Brumback, P. Rajasethupathy, C. Mclntyre, L. Schlachter, N. Suthana, C. Kubu, L. R. Sankary, K. Herrera-Ferrá, S. Goetz, B. Cheeran, G. K. Steinke, C. Hess, L. Almeida, W. Deeb, K. D. Foote, M. S. Okun, Proceedings of the Seventh Annual Deep Brain Stimulation Think Tank: Advances in Neurophysiology, Adaptive DBS, Virtual Reality, Neuroethics and Technology, Front. Hum. Neurosci. 14 (2020), doi:10.3389/fnhum.2020.00054.

21. R. Gilron, S. Little, R. Perrone, R. Wilt, C. De Hemptinne, S. Maria, C. A. Racine, S. Wang, J. L. Ostrem, P. S. Larson, D. Doris, N. B. Galifianakis, I. Bledsoe, M. S. Luciano, H. E. Dawes, A. Gregory, V. Kremen, D. Borton, T. Denison, P. A. Starr, Chronic wireless streaming of invasive neural recordings at home for circuit discovery and adaptive stimulation, bioarxiv (2020), doi:doi: https://doi.org/10.1101/2020.02.13.948349. 22. S. Stanslaski, J. Herron, E. Fehrmann, R. Corey, H. Orser, E. Opri, V. Kremen, B. Brinkmann, A. Gunduz, K. Foote, G. Worrell, T. Denison, in 2018 IEEE International Solid State Circuits Conference - (ISSCC), (IEEE, 2018), pp. 460-462.

23. V. Kremen, B. H. Brinkmann, I. Kim, H. Guragain, M. Nasseri, A. L. Magee, T. Pal Attia, P. Nejedly, V. Sladky, N. Nelson, S.-Y. Chang, J. A. Herron, T. Adamski, S. Baldassano, J. Cimbalnik, V. Vasoli, E. Fehrmann, T. Chouinard, E. E. Patterson, B. Litt, M. Stead, J. Van Gompel, B. K. Sturges, H. J. Jo, C. M. Crowe, T. Denison, G. A. Worrell, Integrating Brain Implants With Local and Distributed Computing Devices: A Next Generation Epilepsy Management System, IEEE J. Transl. Eng. Heal. Med. 6, 1-12 (2018).

24. D. A. Borton, H. E. Dawes, G. A. Worrell, P. A. Starr, T. J. Denison, Developing Collaborative Platforms to Advance Neurotechnology and Its TranslationNeuron (2020), doi:10.1016/j.neuron.2020.10.001.

25. V. Kremen, J. J. Duque, B. H. Brinkmann, B. M. Berry, M. T. Kucewicz, F. Khadjevand, J. Van Gompel, M. Stead, E. K. St Louis, G. A. Worrell, Behavioral state classification in epileptic brain using intracranial electrophysiology, J. Neural Eng. 14, 026001 (2017).

26. V. Kremen, B. H. Brinkmann, J. J. Van Gompel, M. Stead, E. K. St Louis, G. A. Worrell, Automated unsupervised behavioral state classification using intracranial electrophysiology, J. Neural Eng. 16, 026004 (2019).

27. A. Wauquier, J. L. Verheyen, W. A. E. Van Den Broeck, P. A. J. Janssen, Visual and computer-based analysis of $24 \mathrm{~h}$ sleep-waking patterns in the dog, Electroencephalogr. Clin. Neurophysiol. 46, 33-48 (1979).

28. R. Bódizs, A. Kis, M. Gácsi, J. Topál, Sleep in the dog: comparative, behavioral and translational relevance, Curr. Opin. Behav. Sci. 33, 25-33 (2020).

29. IBER, C., The AASM Manual for the Scoring of Sleep and Associated Events: Rules, Terminol. Tech. Specif. (2007) (available at http://ci.nii.ac.jp/naid/10024500923/en/). 30. I. Osorio, The NeuroPace trial: Missing knowledge and insights, Epilepsia 55, 14691470 (2014).

31. M. J. Morrell, In response: The RNS System multicenter randomized double-blinded controlled trial of responsive cortical stimulation for adjunctive treatment of intractable partial epilepsy: Knowledge and insights gained, Epilepsia 55, 1470-1471 (2014). 32. S. Hochreiter, J. Schmidhuber, Long Short-Term Memory, Neural Comput. 9, 1735- 
1780 (1997).

33. Y. LeCun, Y. Bengio, in The handbook of brain theory and neural networks, Michael A. Arbib, Ed. (MIT Press, Cambridge, MA, USA, 1998), pp. 255-258.

34. K. A. Davis, B. K. Sturges, C. H. Vite, V. Ruedebusch, G. Worrell, A. B. Gardner, K. Leyde, W. D. Sheffield, B. Litt, A novel implanted device to wirelessly record and analyze continuous intracranial canine EEG, Epilepsy Res. 96, 116-122 (2011).

35. B. H. Brinkmann, J. Wagenaar, D. Abbot, P. Adkins, S. C. Bosshard, M. Chen, Q. M. Tieng, J. He, F. J. Muñoz-Almaraz, P. Botella-Rocamora, J. Pardo, F. Zamora-Martinez, M. Hills, W. Wu, I. Korshunova, W. Cukierski, C. Vite, E. E. Patterson, B. Litt, G. A. Worrell, Crowdsourcing reproducible seizure forecasting in human and canine epilepsy, Brain 139, 1713-1722 (2016).

36. M. J. Cook, T. J. O’Brien, S. F. Berkovic, M. Murphy, A. Morokoff, G. Fabinyi, W. D’Souza, R. Yerra, J. Archer, L. Litewka, S. Hosking, P. Lightfoot, V. Ruedebusch, W. D. Sheffield, D. Snyder, K. Leyde, D. Himes, Prediction of seizure likelihood with a long-term, implanted seizure advisory system in patients with drug-resistant epilepsy: A first-in-man study, Lancet Neurol. 12, 563-571 (2013).

37. S. Baldassano, X. Zhao, B. Brinkmann, V. Kremen, J. Bernabei, M. Cook, T. Denison, G. Worrell, B. Litt, Cloud computing for seizure detection in implanted neural devices, J. Neural Eng. 16, 026016 (2019).

38. J. Engel, T. A. Pedley, J. Aicardi, M. A. Dicter, P. Emilio, Epilepsy: A Comprehensive Textbook (Lippincott Williams \& Wilkins (LWW), ed. 2nd, 2007).

39. P. J. Karoly, D. R. Freestone, R. Boston, D. B. Grayden, D. Himes, K. Leyde, U. Seneviratne, S. Berkovic, T. O'Brien, M. J. Cook, Interictal spikes and epileptic seizures: their relationship and underlying rhythmicity, Brain 139, 1066-1078 (2016).

40. M. O. Baud, J. K. Kleen, E. A. Mirro, J. C. Andrechak, D. King-Stephens, E. F. Chang, V. R. Rao, Multi-day rhythms modulate seizure risk in epilepsy, Nat. Commun. 9, 88 (2018).

41. N. M. Gregg, M. Nasseri, V. Kremen, E. E. Patterson, B. K. Sturges, T. J. Denison, B. H. Brinkmann, G. A. Worrell, Circadian and multiday seizure periodicities, and seizure clusters in canine epilepsy, Brain Commun. 2 (2020), doi:10.1093/braincomms/fcaa008.

42. R. Janca, P. Jezdik, R. Cmejla, M. Tomasek, G. A. Worrell, M. Stead, J. Wagenaar, J. G. R. Jefferys, P. Krsek, V. Komarek, P. Jiruska, P. Marusic, Detection of Interictal Epileptiform Discharges Using Signal Envelope Distribution Modelling: Application to Epileptic and Non-Epileptic Intracranial Recordings, Brain Topogr. 28, 172-183 (2014). 43. S. Järvenpää, J. Peltola, S. Rainesalo, E. Leinonen, K. Lehtimäki, K. Järventausta, Reversible psychiatric adverse effects related to deep brain stimulation of the anterior thalamus in patients with refractory epilepsy, Epilepsy Behav. 88, 373-379 (2018). 44. B. R. Voges, F. C. Schmitt, W. Hamel, P. M. House, C. Kluge, C. K. E. Moll, S. R. Stodieck, Deep brain stimulation of anterior nucleus thalami disrupts sleep in epilepsy patients, Epilepsia 56, e99-e103 (2015).

45. R. S. Fisher, A. L. Velasco, Electrical brain stimulation for epilepsy, Nat. Rev. Neurol. 10, 261-270 (2014).

46. J. Yamamoto, A. Ikeda, M. Kinoshita, R. Matsumoto, T. Satow, K. Takeshita, M. Matsuhashi, N. Mikuni, S. Miyamoto, N. Hashimoto, H. Shibasaki, Low-frequency electric cortical stimulation decreases interictal and ictal activity in human epilepsy., Seizure 15, 520-527 (2006).

47. M. Kinoshita, A. Ikeda, M. Matsuhashi, R. Matsumoto, T. Hitomi, T. Begum, K. Usui, M. Takayama, N. Mikuni, S. Miyamoto, N. Hashimoto, H. Shibasaki, Electric cortical 
stimulation suppresses epileptic and background activities in neocortical epilepsy and mesial temporal lobe epilepsy, Clin. Neurophysiol. 116, 1291-1299 (2005).

48. T. A. Wozny, W. J. Lipski, A. Alhourani, E. D. Kondylis, A. Antony, R. M. Richardson, Effects of hippocampal low-frequency stimulation in idiopathic non-human primate epilepsy assessed via a remote-sensing-enabled neurostimulator, Exp. Neurol. (2017), doi:10.1016/j.expneurol.2017.05.003.

49. L. Velíšek, J. Velíšková, P. K. Stanton, Low-frequency stimulation of the kindling focus delays basolateral amygdala kindling in immature rats, Neurosci. Lett. (2002),

doi:10.1016/S0304-3940(02)00294-X.

50. J. H. Goodman, R. E. Berger, T. K. Tcheng, Preemptive low-frequency stimulation decreases the incidence of amygdala-kindled seizures, Epilepsia (2005), doi:10.1111/j.0013-9580.2005.03804.x.

51. K. B. Kile, N. Tian, D. M. Durand, Low frequency stimulation decreases seizure activity in a mutation model of epilepsy, Epilepsia (2010), doi:10.1111/j.1528-1167.2010.02679.x.

52. S. Rashid, G. Pho, M. Czigler, M. A. Werz, D. M. Durand, Low frequency stimulation of ventral hippocampal commissures reduces seizures in a rat model of chronic temporal lobe epilepsy, Epilepsia (2012), doi:10.1111/j.1528-1167.2011.03348.x.

53. Y. Tang, D. M. Durand, A novel electrical stimulation paradigm for the suppression of epileptiform activity in an in vivo model of mesial temporal lobe status epilepticus, Int. J. Neural Syst. (2012), doi:10.1142/S0129065712500062.

54. E. Paschen, C. Elgueta, K. Heining, D. M. Vieira, P. Kleis, C. Orcinha, U. Häussler, M. Bartos, U. Egert, P. Janz, C. A. Haas, Hippocampal low-frequency stimulation prevents seizure generation in a mouse model of mesial temporal lobe epilepsy, Elife (2020), doi:10.7554/ELIFE.54518.

55. C. E. Elger, F. Mormann, Seizure prediction and documentation-two important problems, Lancet Neurol. 12, 531-532 (2013).

56. S. T. Herman, T. S. Walczak, C. W. Bazil, Distribution of partial seizures during the sleep-wake cycle: Differences by seizure onset site, Neurology 56, 1453-1459 (2001). 57. C. W. Bazil, T. S. Walczak, Effects of Sleep and Sleep Stage on Epileptic and Nonepileptic Seizures, Epilepsia 38, 56-62 (1997).

58. C. W. Bazil, Epilepsy and sleep disturbance, Epilepsy Behav. 4, 39-45 (2003). 59. M. R. Bower, M. Stead, R. S. Bower, M. T. Kucewicz, V. Sulc, J. Cimbalnik, B. H. Brinkmann, V. M. Vasoli, E. K. St. Louis, F. B. Meyer, W. R. Marsh, G. A. Worrell, Evidence for Consolidation of Neuronal Assemblies after Seizures in Humans, J. Neurosci. 35, 999-1010 (2015).

60. M. R. Bower, M. T. Kucewicz, E. K. St. Louis, F. B. Meyer, W. R. Marsh, M. Stead, G. A. Worrell, Reactivation of seizure-related changes to interictal spike shape and synchrony during postseizure sleep in patients, Epilepsia 58, 94-104 (2017).

61. P. Nejedly, V. Kremen, V. Sladky, M. Nasseri, H. Guragain, P. Klimes, J. Cimbalnik, Y. Varatharajah, B. H. Brinkmann, G. A. Worrell, Deep-learning for seizure forecasting in canines with epilepsy, J. Neural Eng. 16, 036031 (2019).

62. S. N. Baldassano, B. H. Brinkmann, H. Ung, T. Blevins, E. C. Conrad, K. Leyde, M. J. Cook, A. N. Khambhati, J. B. Wagenaar, G. A. Worrell, B. Litt, Crowdsourcing seizure detection: Algorithm development and validation on human implanted device recordings, Brain 140, 1680-1691 (2017).

63. L. Kuhlmann, P. Karoly, D. R. Freestone, B. H. Brinkmann, A. Temko, A. Barachant, F. Li, G. Titericz, B. W. Lang, D. Lavery, K. Roman, D. Broadhead, S. Dobson, G. Jones, Q. Tang, I. Ivanenko, O. Panichev, T. Proix, M. Náhlík, D. B. Grunberg, C. Reuben, G. 
Worrell, B. Litt, D. T. J. Liley, D. B. Grayden, M. J. Cook, Epilepsyecosystem.org: crowdsourcing reproducible seizure prediction with long-term human intracranial EEG, Brain (2018), doi:10.1093/brain/awy210.

64. H. Potschka, Animal and human data: where are our concepts for drug-resistant epilepsy going? Epilepsia (2013), doi:10.1111/epi.12181.

65. J. J. Van Gompel, B. T. Klassen, G. A. Worrell, K. H. Lee, C. Shin, C. Z. Zhao, D. A. Brown, S. J. Goerss, B. A. Kall, M. Stead, Anterior nuclear deep brain stimulation guided by concordant hippocampal recording, Neurosurg. Focus 38, E9 (2015).

66. S. S. Grewal, E. H. Middlebrooks, T. J. Kaufmann, M. Stead, B. N. Lundstrom, G. A. Worrell, C. Lin, S. Baydin, J. J. Van Gompel, Fast gray matter acquisition T1 inversion recovery $\mathrm{MRI}$ to delineate the mammillothalamic tract for preoperative direct targeting of the anterior nucleus of the thalamus for deep brain stimulation in epilepsy, Neurosurg. Focus 45, E6 (2018).

67. V. Gerla, V. Kremen, M. Macas, D. Dudysova, A. Mladek, P. Sos, L. Lhotska, Iterative expert-in-the-loop classification of sleep PSG recordings using a hierarchical clustering, $J$. Neurosci. Methods 317, 61-70 (2019).

68. M. Ghassemi, B. Moody, L. Lehman, C. Song, Q. Li, H. Sun, B. Westover, G. Clifford, in (2018).

69. A. Burrello, K. Schindler, L. Benini, A. Rahimi, in 2018 IEEE Biomedical Circuits and Systems Conference, BioCAS 2018 - Proceedings, (Institute of Electrical and Electronics Engineers Inc., 2018).

70. A. Kharbouch, A. Shoeb, J. Guttag, S. S. Cash, An algorithm for seizure onset detection using intracranial EEG, Epilepsy Behav. 22, S29-S35 (2011).

71. N. D. Truong, L. Kuhlmann, M. R. Bonyadi, J. Yang, A. Faulks, O. Kavehei, Supervised learning in automatic channel selection for epileptic seizure detection, Expert Syst. Appl. 86, 199-207 (2017).

72. P. Nejedly, V. Kremen, V. Sladky, J. Cimbalnik, P. Klimes, F. Plesinger, I. Viscor, M. Pail, J. Halamek, B. H. Brinkmann, M. Brazdil, P. Jurak, G. Worrell, Exploiting Graphoelements and Convolutional Neural Networks with Long Short Term Memory for Classification of the Human Electroencephalogram, Sci. Rep. 9, 11383 (2019).

73. R. R. Clancy, A. Legido, The Exact Ictal and Interictal Duration of Electroencephalographic Neonatal Seizures, Epilepsia 28, 537-541 (1987). 


\section{MH1}

\begin{tabular}{|c|c|c|c|}
\hline & F1 Score & Kappa & Accuracy \\
\hline Wake & 0.98 & 0.97 & 0.99 \\
\hline N2 & 0.42 & 0.32 & 0.82 \\
\hline N3 & 0.76 & 0.65 & 0.85 \\
\hline N2+N3 & 0.96 & 0.92 & 0.95 \\
\hline REM & 0.87 & 0.85 & 0.96 \\
\hline Overall & 0.79 & 0.73 & 0.81 \\
\hline
\end{tabular}

\section{MD2}

\begin{tabular}{|c|c|c|c|}
\hline & F1 Score & Kappa & Accuracy \\
\hline Wake & 0.84 & 0.82 & 0.96 \\
\hline N2 & 0.77 & 0.69 & 0.88 \\
\hline N3 & 0.94 & 0.89 & 0.95 \\
\hline N2+N3 & 0.93 & 0.85 & 0.92 \\
\hline REM & 0.89 & 0.86 & 0.95 \\
\hline Overall & 0.87 & 0.82 & 0.87 \\
\hline
\end{tabular}

\begin{tabular}{|c|c|c|c|}
\hline & F1 Score & Kappa & Accuracy \\
\hline Wake & 0.93 & 0.88 & 0.94 \\
\hline N2 & 0.47 & 0.39 & 0.86 \\
\hline N3 & 0.87 & 0.81 & 0.92 \\
\hline N2+N3 & 0.90 & 0.80 & 0.88 \\
\hline REM & 0.66 & 0.62 & 0.93 \\
\hline Overall & 0.83 & 0.74 & 0.83 \\
\hline
\end{tabular}

Table 1. The performance of automated behavioral state classification. Results for wake, nonrapid eye movement (NREM 2, 3), and rapid eye movement (REM) from a human (MH1) and three dogs (MD1, MD2, and MD3). Simultaneous polysomnograms and intracranial EEG data were collected in the hospital over multiple nights. Expert visual review of video and polysomnograms were used to create gold standard sleep classification catalogues for training, validation and testing of algorithms (see methods). 


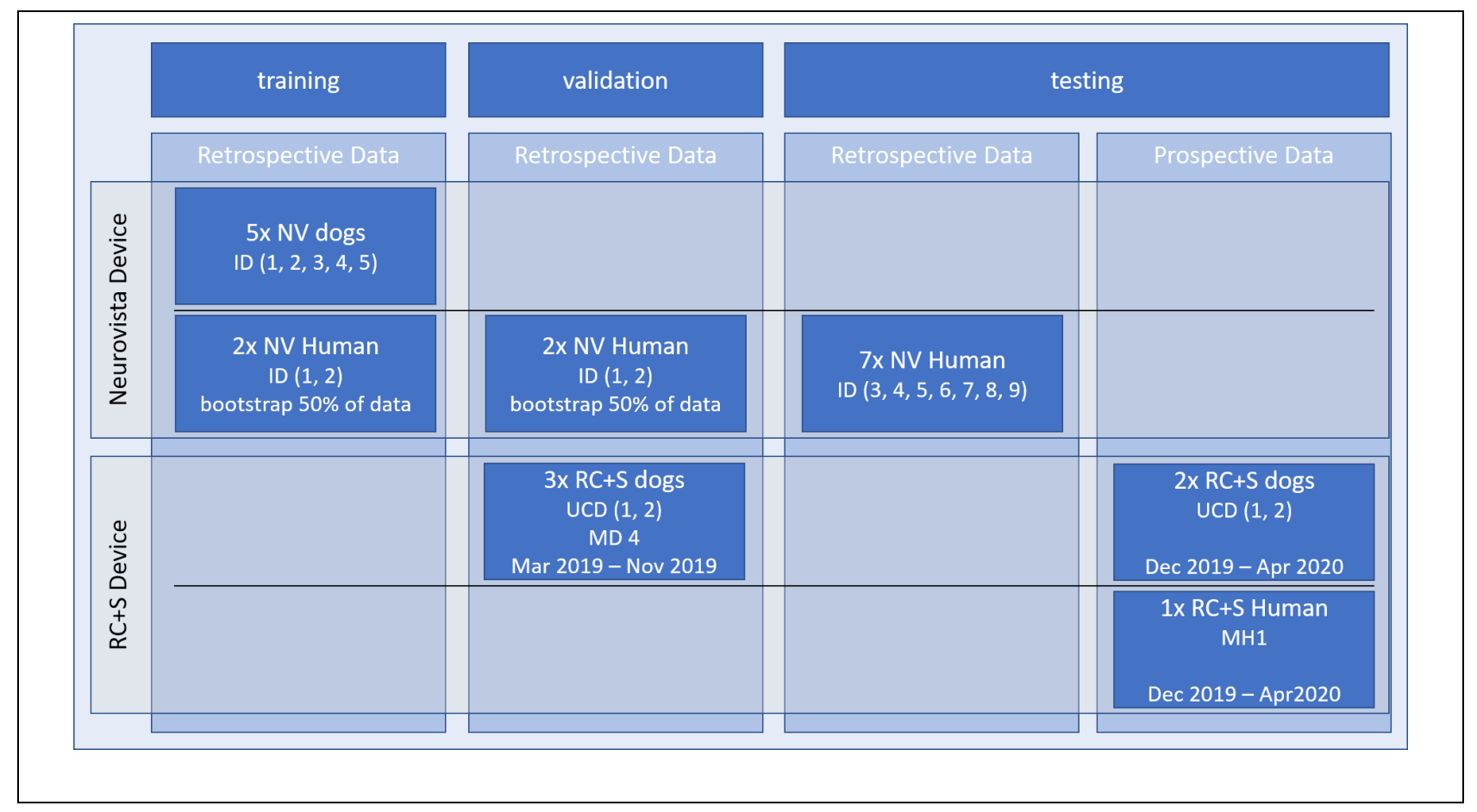

Table 2. Seizure detection datasets. Training, validation, and testing data used in development of a generic, automated seizure detection algorithm for canines and humans. Retrospective data included human and canine datasets acquired with two different investigational devices, Neurovista $(\mathrm{NV})$ and $\mathrm{RC}+\mathrm{S}^{\mathrm{TM}}$ device. Algorithm training was performed using retrospective data from humans and canines collected with NV devices. The validation data used NV data from two humans $(\mathrm{N} 1,2)$ and $R C+S^{T M}$ data from three canines (UCD 1, 2; MD4). Pseudo-prospective (NV data from 7 humans; N3-9) and prospective $\left(R C+S^{T M}\right.$ data from $M H 1$ and 2 pet dogs UCD1, 2) ambulatory testing in human and canines in accustomed settings (human living at home and two pet dogs living with their owners) was performed over multiple months. 


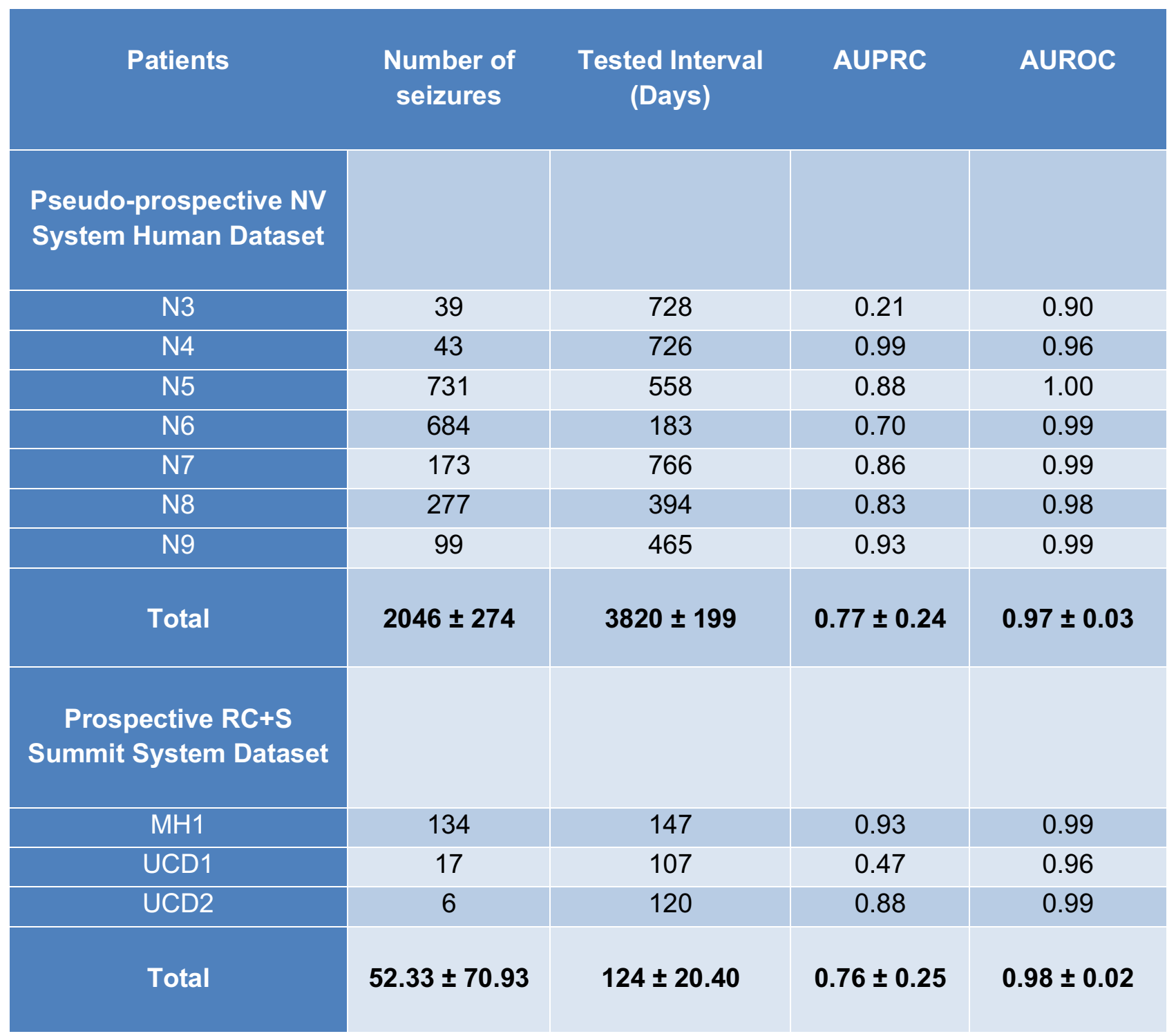

Table 3. Seizure detection results. Performance of the generalizable seizure detection model for canine and human seizures deployed on multiple out-of-sample datasets both for pseudo-prospective NeuroVista (NV) and fully ambulatory prospective $\mathrm{RC}+\mathrm{S}^{\mathrm{TM}}$ testing (chronically monitored: $\mathrm{MH} 1$ - human subject, UCD1,2 - dog subjects). Standard machine learning performance metrics are shown together with the number of seizures and number of recording days in the datasets (AUPRC - area under precision recall curve; AUROC - area under receiver operating characteristic curve). 


\begin{tabular}{|c|c|c|c|c|}
\hline Characteristics & Cluster & Non-Cluster & Awake/Day & Night \\
\hline IES rate per minute & $37.4 \pm 29.1$ & $17.9 \pm 7.2$ & $13.8 \pm 8.7$ & $41.6 \pm 23.7$ \\
\hline F1 - score & $0.84 \pm 0.09$ & $0.8 \pm 0.05$ & $0.81 \pm 0.08$ & $0.82 \pm 0.08$ \\
\hline PPV & $0.81 \pm 0.2$ & $0.74 \pm 0.14$ & $0.71 \pm 0.12$ & $0.8 \pm 0.18$ \\
\hline Sensitivity & $0.9 \pm 0.08$ & $0.9 \pm 0.08$ & $0.94 \pm 0.001$ & $0.89 \pm 0.09$ \\
\hline
\end{tabular}

Table 4. Interictal epileptiform spike (IES) rates. Results from automated classifier for human patient under different conditions and compared to expert review. The F1-score comparing the automated and expert visual review was similar for each condition studied.

\begin{tabular}{|c|c|c|c|c|c|c|}
\hline & $\begin{array}{c}\text { Biomarkers } \\
\& \\
\text { Brain States }\end{array}$ & $\begin{array}{c}\text { N2 } \\
\text { Hours }\end{array}$ & $\begin{array}{l}\text { N3 } \\
\text { Hours }\end{array}$ & $\begin{array}{l}\mathrm{N} 2+\mathrm{N} 3 \\
\text { Hours }\end{array}$ & $\begin{array}{l}\text { REM } \\
\text { Hours }\end{array}$ & $\begin{array}{c}\text { Sleep Time } \\
\text { Hours }\end{array}$ \\
\hline \multirow{4}{*}{ 폴 } & $\begin{array}{c}\text { No EBS } \\
\text { (22 nights) }\end{array}$ & $0.59 \pm 0.12$ & $3.87 \pm 0.51$ & $4.46 \pm 0.55$ & $2.91 \pm 0.63$ & $7.37 \pm 0.85$ \\
\hline & $\begin{array}{l}\text { LF EBS } \\
\text { (84 nights) }\end{array}$ & $0.70 \pm 0.19^{* *}$ & $3.77 \pm 0.48$ & $4.47 \pm 0.50$ & $1.74 \pm 0.72^{* \star}$ & $6.21 \pm 0.99^{* *}$ \\
\hline & $\begin{array}{l}\text { HF EBS } \\
\text { (63 nights) }\end{array}$ & $0.72 \pm 0.18^{* *}$ & $3.98 \pm 0.62$ & $4.71 \pm 0.5^{*}$ & $2.28 \pm 0.63^{* *}$ & $6.98 \pm 0.95^{*}$ \\
\hline & $\begin{array}{c}\text { Continuous LF } \\
\text { \& Responsive } \\
\text { HF EBS } \\
\text { (22 nights) }\end{array}$ & $1.01 \pm 0.29^{* *}$ & $3.16 \pm 0.50^{* *}$ & $4.17 \pm 0.4^{*}$ & $2.02 \pm 1.06^{* \star}$ & $6.20 \pm 1.17^{* *}$ \\
\hline
\end{tabular}

Table 5. Influence of electrical brain stimulation (EBS) on sleep length. The sleep length of MH1 subject during long-term monitoring. Both, continuous low frequency (LF), high frequency (HF) and adaptive stimulation with continuous LF and responsive HF stimulation during wake significantly reduced total sleep time and length of rapid eye-movement (REM) sleep and prolonged the N2 phase of sleep compared to baseline with no stimulation $\left({ }^{* *} p<0.01 ;{ }^{*} p<0.05\right)$. All nights analyzed had data transmission rates $>85 \%$ to ensure results were not biased by iEEG data telemetry drops. 


\begin{tabular}{|c|c|c|c|c|c|c|c|}
\hline \multicolumn{2}{|c|}{$\begin{array}{l}\text { Biomarkers } \\
\text { \&. Brain States }\end{array}$} & $\begin{array}{l}\text { Days of } \\
\text { monitoring }\end{array}$ & $\begin{array}{c}\text { Data } \\
\text { Rate[\%] }\end{array}$ & $\begin{array}{l}\text { Reported } \\
\text { Seizures }\end{array}$ & $\begin{array}{l}\text { Confirmed } \\
\text { Seizures }\end{array}$ & $\begin{array}{c}\text { Seizures } \\
\text { (per daily) }\end{array}$ & $\begin{array}{l}\text { IES rates } \\
\text { (per } \mathrm{min})\end{array}$ \\
\hline \multirow{4}{*}{ 폴 } & No EBS & 30 & 72 & 10 & 27 & $0.90 \pm 0.94$ & $37.65 \pm 6.29$ \\
\hline & LF EBS & 186 & 72 & 77 & 171 & $0.92 \pm 1.05$ & $29.46 \pm 7.59^{* *}$ \\
\hline & HF EBS & 94 & 73 & 46 & 113 & $1.20 \pm 1.48$ & $33.19 \pm 8.44^{* *}$ \\
\hline & $\begin{array}{c}\text { Continuous LF } \\
\text { \& Responsive } \\
\text { HF EBS }\end{array}$ & 28 & 72 & 2 & 20 & $0.71 \pm 0.75$ & $35.42 \pm 4.85^{*}$ \\
\hline \multirow{3}{*}{ 억 } & No EBS & 79 & 58 & 12 & 23 & $0.29 \pm 0.64$ & $73.30 \pm 32.57$ \\
\hline & LF EBS & 59 & 41 & 14 & 13 & $0.22 \pm 0.94^{* *}$ & $56.66 \pm 23.31^{* *}$ \\
\hline & HF EBS & 274 & 36 & 2 & 73 & $0.27 \pm 1.50^{* *}$ & $106.18 \pm 57.25^{\star \star}$ \\
\hline \multirow{2}{*}{ 今ั } & No EBS & 186 & 59 & 8 & 19 & $0.10 \pm 0.40$ & $228.95 \pm 75.25$ \\
\hline & LF EBS & 98 & 49 & 7 & 19 & $0.20 \pm 0.97$ & $217.22 \pm 75.43^{*}$ \\
\hline
\end{tabular}

Table 6. Influence of electrical brain stimulation (EBS) on seizure rates and interictal epileptiform spike (IES) rates. Results in human (MH1) and two pet dogs (UCD1, UCD2) chronically monitored in their natural environments. Continuous low frequency (LF) EBS reduced IES in all subjects (MH1, UCD1, UCD2) compared to no EBS, but only reduced seizures in UCD1. Continuous high frequency (HF) EBS had a variable effect on IES rates, decreasing IES rates in $\mathrm{MH} 1$ but increasing IES rates in UCD1, and decreasing seizures in UCD1 to a smaller degree than LF EBS. Because of the pet dogs brittle epilepsy HF EBS was not explored in UCD2. Lastly, adaptive circadian continuous LF stimulation combined with responsive HF EBS during waking hours was trialed in the human subject because the majority of her seizures occurred with a circadian pattern in late afternoon, and HF EBS was poorly tolerated during sleep. The circadian EBS trial reduced IES and had a trend towards lower seizure counts.

Acknowledgments: We thank the people with epilepsy and the pet owners for participating in this research. We appreciate the technical and patient-focused support provided by Cindy Nelson and Karla Crockett. We thank Certicon a.s. for use of CyberPSG tool.

Funding: This work was financially supported primarily by the National Institutes of Health (NIH):

NIH Brain Initiative UH2/UH3NS95495

NIH Brain Initiative R01-NS09288203

In part by:

Mayo Clinic Benefactors

Institutional support at Czech Technical University in Prague, Prague, Czech Republic 
European Regional Development Fund-Project ENOCH (No.CZ.02.1.01/0.0/0.0/16_019/0000868) Ministry of Education, Youth and Sports of the Czech Republic project no. LTAUSA 18056 (program INTER-EXCELLENCE)

Sleep analysis was supported in part by DARPA project HR0011-20-2-0028

This work benefited from the community expertise and resources made available by the NIH Open Mind Consortium NIH U24-NS113637 (https://openmind-consortium.github.io/)

IB, VM, and LW were supported by the Mayo Clinic Graduate School of Biomedical Sciences

\section{Author contributions:}

Conceptualization: VS, PN, BB, KL, TD, GW, and VK

Animal experiments: BB, IK, CC, BS, JVG, FM, GW, and VK

Data processing and analysis-electrophysiology: VS, PN, FM, BB, RJ, JC, TA, DC, IB, VM, LW, GW, VK

Clinical data: ESL, NG, BL, KM, KL, MK, JVG, GW

Writing-original draft: VS, PN, GW and VK

Writing, review, and editing: all authors.

Competing interests: GW, VK and BB declare intellectual property related to behavioral state classification analytics. GW, BB, and BNL declare intellectual property licensed to Cadence Neuroscience Inc. GW has licensed intellectual property to NeuroOne, Inc. GW and BNL are investigators for the Medtronic Deep Brain Stimulation Therapy for Epilepsy Post-Approval Study (EPAS). VM is a paid summer intern at Medtronic. GW previously assisted in a Mayo Clinic Medtronic sponsored FDA-IDE for the investigational Medtronic Activa PC+S device. The remaining authors declare that they have no competing interests. The investigational Medtronic Summit $R C+S^{T M}$ devices used in this research were provided free of charge as part of NIH Brain Initiative UH2/UH3-NS95495.

Data and materials availability: All results associated with this study are present in the paper.

The raw data are available upon reasonable request. 